\title{
Roots of the Canonical Bundle of the Universal Teichmiiller Curve and Certain Subgroups of the Mapping Class Group
}

\author{
Patricia L. Sipe \\ Department of Mathematics, University of Michigan, Ann Arbor, MI 48109, USA
}

\section{Introduction and Statement of Results}

1.1. Throughout this paper, let $S$ be a fixed, smooth $\left(C^{\infty}\right)$ orientable closed surface of genus $g \geqq 2$. Let $S$ be equipped with a preferred complex structure; we denote the Riemann surface by $X_{0}$, and the Teichmüller space of $X_{0}$ by $T_{g}$. There is a Fuchsian group $\Gamma_{g}$ operating in the unit disk so that $X_{0}=U / \Gamma_{g}$.

The universal Teichmüller curve $V_{g}$ is a fibre space over Teichmuiller space, with $X_{t}$, the fibre over $t \in T_{q}$ conformally equivalent to the Riemann surface represented by $t \in T_{g}$. (Each $X_{t}$ is diffeomorphic to $S$.) $V_{g}$ is a complex manifold of dimension $3 g-2$, and we denote its canonical line bundle by $K\left(V_{g}\right)$. We define $L$, a holomorphic complex line bundle over $V_{g}$, to be an $n^{\text {th }}$ root of $K\left(V_{g}\right)$ iff $L^{\otimes n} \simeq K\left(V_{g}\right)$. The purpose of this paper is to study the $n^{\text {th }}$ roots of $K\left(V_{g}\right)$; in particular, we investigate an action of the Teichmüller modular group $\operatorname{Mod}\left(\Gamma_{g}\right)(=$ mapping class group) on the (finite) set of $n^{\text {th }}$ roots.

Denote the tangent and cotangent bundles of a manifold $M$ by $T(M)$ and $T^{*}(M)$, respectively; $T_{0}(M)$ and $T_{0}^{*}(M)$ denote the corresponding bundles with their zero sections removed. David Mumford observed (informal communication) that over a single Riemann surface $X$, the $n^{\text {th }}$ roots correspond to certain homomorphisms $\lambda: H_{1}\left(T_{0}(X), \mathbb{Z}_{n}\right) \rightarrow \mathbb{Z}_{n}$. He suggested trying to work out the action of $\operatorname{Mod}\left(\Gamma_{g}\right)$ in terms of its action on these homomorphisms.

1.2. Let $f: S \rightarrow S$ be a diffeomorphism, and $\hat{f}$ its equivalence class in $\operatorname{Mod}\left(\Gamma_{g}\right)$. $\operatorname{Mod}\left(\Gamma_{g}\right)$ acts on $V_{g}$ as a group of biholomorphic maps, and that action induces (by pullback) an action on the set of holomorphic complex line bundles which are $\boldsymbol{n}^{\text {th }}$ roots of $K\left(V_{g}\right)$. The $n^{\text {th }}$ roots are a finite set of order $n^{2 g}$. The action of $\operatorname{Mod}\left(\Gamma_{g}\right)$ on that set gives a homomorphism $\operatorname{Mod}\left(\Gamma_{g}\right) \rightarrow \operatorname{Perm}\left(n^{2 g}\right)$, where $\operatorname{Perm}\left(n^{2 g}\right)$ is the permutation group on a set of order $n^{2 g}$.

Let $G_{g, n}$ denote the kernel of that homomorphism, that is, the subgroup of $\operatorname{Mod}\left(\Gamma_{g}\right)$ which acts trivially on all $n^{\text {th }}$ roots. These groups are of particular interest, because they are normal subgroups of finite index in $\operatorname{Mod}\left(\Gamma_{g}\right)$. The study of the action of $\operatorname{Mod}\left(\Gamma_{g}\right)$ on $n^{\text {th }}$ roots leads to the following characterization of these subgroups: 
Theorem A. The subgroup $G_{g, n}$ of $\operatorname{Mod}\left(\Gamma_{g}\right)$ which leaves every $n^{\text {th }}$ root fixed is precisely the subgroup of elements which induce the identity on the homology $(\bmod n)$ of the unit tangent bundle of $S$.

Notice that $H_{1}\left(T_{0}(S), \mathbb{Z}_{n}\right)$ is the homology of the unit tangent bundle of $S(\bmod n) ;$ let $\alpha$ denote the loop around the origin in the fibre. Denote

$$
\Lambda\left(T_{0}(S)\right)=\left\{\text { homomorphisms } \lambda: H_{1}\left(T_{0}(S), \mathbb{Z}_{n}\right) \rightarrow \mathbb{Z}_{w} \text {, with } \lambda(\alpha)=-1\right\}
$$

$\operatorname{Mod}\left(\Gamma_{g}\right)$ acts on $A\left(T_{0}(S)\right)$ [the differential of $\hat{f} \in \operatorname{Mod}\left(\Gamma_{g}\right)$ induces a map on homology, and composition of $\lambda$ with this map gives a new element of $\Lambda\left(T_{0}(S)\right]$. Comparison of this action with the action of $n^{\text {th }}$ roots leads to a proof of Theorem A. We establish (Theorem 1) a bijection between the set of $n^{\text {th }}$ roots and $\Lambda\left(T_{0}(S)\right)$; we then prove that the actions of $\operatorname{Mod}\left(\Gamma_{q}\right)$ on those two sets correspond under that identification. These matters are discussed in Sect. 2, although the proof of Theorem 1 is deferred to Sects. 5 and 6.

Studying the $n^{\text {th }}$ roots as homomorphisms allows us to use geometric methods to prove

Theorem B. The action of $\operatorname{Mod}\left(\Gamma_{g}\right)$ on $n^{\text {th }}$ roots (described as certain homomorphisms: $H_{1}\left(T_{0}(S), \mathbb{Z}_{n}\right) \rightarrow \mathbb{Z}_{n}$ can be computed for a set of generators for $\operatorname{Mod}\left(\Gamma_{g}\right)$.

The indicated computation is carried out in Sect. 3 .

Theorem A implies that the image of $G_{g, n}$ in the symplectic group is contained in the congruence subgroup of rank $n$. In Sect. 4 , we use a theorem of Mennicke to show that the opposite containment holds, thereby obtaining

Theorem $\mathbf{C}$. The image of $G_{g, n}$ under the mapping $\varrho: \operatorname{Mod}\left(\Gamma_{g}\right) \rightarrow \operatorname{Sp}(g, \mathbb{Z})$ is precisely the congruence subgroup of rank $n$.

\section{The Actions of the Modular Group (Theorem A)}

2.1. The reader of this paper should be familiar with the definitions and basic properties of the Teichmüller space, the universal Teichmüller curve, and the Teichmüller Modular (= Mapping class) group. We summarize here only the material necessary to establish our notation, which follows [6] very closely. Other references for the necessary background material and further details are [2-4].

The Teichmüller space $T_{g}$ is a complex manifold of dimension $3 g-3$ and can be identified with a bounded open set in $\mathbb{C}^{3 g-3}$. The Bers Fibre space $F_{g}$ is a certain subset of $T_{g} \times \mathbb{C} \simeq \mathbb{C}^{3 g-2}$. The Fuchsian group $\Gamma_{g}$ acts on $F_{g}$ as a group of biholomorphic maps, producing the universal Teichmüller curve as the quotient $V_{g}=F_{g} / T_{g}[2]$.

Recall that a holomorphic family of Riemann surfaces over $B$ consists of a pair of connected complex manifolds $V$ and $B$ and a proper holomorphic submersion $\pi$ mapping $V$ onto $B$ where $X_{t}=\pi^{-1}(t)$ is a closed Riemann surface of genus $g \geqq 2$ for each $t \in B$. The Universal Teichmüller curve $V_{g}$ is a holomorphic family of Riemann surfaces over $T_{g}$.

While the complex structure on $V_{g}$ is crucial to this study, at times we will use the following proposition to study $V_{g}$ as a $C^{\infty}$ manifold. 
Proposition 2.1. There is a fibre-preserving diffeomorphism $\theta: V_{g} \rightarrow T_{g} \times S$. The restriction of $\theta$ to the fibre $\pi^{-1}(t) \subseteq V_{g}$ is a diffeomorphism $\theta^{t}: X_{t} \rightarrow S$.

Proof. $\pi: V_{g} \rightarrow T_{g}$ is a proper mapping onto $T_{g}$, and therefore defines a (locally trivial) $C^{\infty}$ fibre bundle by Ehresmann's Theorem [7]. Let $t_{0} \in T_{g}$. The local triviality says that there is a neighborhood $U$ of $t_{0}$ with $\pi^{-1}(U)$ diffeomorphic to $U \times \pi^{-1}\left(t_{0}\right)$. Of course, $\pi^{-1}\left(t_{0}\right)$ is a Riemann surface diffeomorphic to $S . T_{\boldsymbol{g}}$ is diffeomorphic to $\mathbb{R}^{6 g-6}$ (see [10]), so it is smoothly contractible, which implies that $V g$ is diffeomorphic to the product $T_{g} \times S$.

2.2. Once we have a holomorphic family of Riemann surfaces, it is natural to study holomorphic families of structures associated with Riemann surfaces, as in $[4,5]$. The basic objects of study here are certain holomorphic families of line bundles over $T_{g}$.

Recall that a holomorphic complex vector bundle of rank $r$ consists of complex manifolds $L$ and $M$ and a complex analytic surjection $\pi: L \rightarrow M$; the fibres $\pi^{-1}(p)$ of the (locally trivial) bundle are complex vector spaces of dimension $r$. We write $L \simeq L^{\prime}$ if two holomorphic complex vector bundles $\pi: L \rightarrow M$ and $\pi^{\prime}: L^{\prime} \rightarrow M$ are holomorphically equivalent (the equivalence $\Phi: L \rightarrow L^{\prime}$ is analytic, and complex linear on fibres). By a line bundle we mean a holomorphic complex vector bundle of rank 1 (or an equivalence class of such bundles). (See [9] and [8] for discussions of vector bundles and line bundles.)

Definition. $A$ holomorphic family of line bundles over $B$ is given by $L \rightarrow V \rightarrow B$ where $V \rightarrow B$ is a holomorphic family of Riemann surfaces and $L \rightarrow V$ is a holomorphic complex line bundle.

Note that the projection $L \rightarrow B$ has the property that the fibre over $t \in B$ is a line bundle $L_{t}$ over $X_{r}$.

Definition. Two holomorphic families of line bundles $L \rightarrow V \rightarrow B$ and $L^{\prime} \rightarrow V \rightarrow B$ are equivalent if and only if the line bundles $L \rightarrow V$ and $L^{\prime} \rightarrow V$ are equivalent as holomorphic complex line bundles.

We denote the holomorphic tangent bundle of a manifold $M$ by $T(M) \rightarrow M$; its transition functions are given by the complex Jacobian matrices of the change of coordinate functions on $M$. Its dual, the cotangent bundle $T^{*}(M) \rightarrow M$ has transition functions which are the transpose of the inverse of those for $T(M)$. The canonical bundle $K(M) \rightarrow M$ is the determinant of the cotangent bundle; that is, it is the holomorphic complex line bundle whose transition functions are the determinant of those of $T^{*}(M)$.

Definition. If $L^{\prime} \rightarrow M$ is a holomorphic complex line bundle over the complex manifold $M$, and $n$ is an integer, an $n^{\text {th }}$ root of $L^{\prime}$ is a line bundle $L$ with $L^{\otimes_{n}} \simeq L^{\prime}$. In particular, an $n^{\text {th }}$ root of the canonical bundle is a bundle $L$ with $L^{\otimes n} \simeq K(M)$.

Remark. Because the transition functions are just nonvanishing holomorphic functions, the (equivalence classes of line bundles form a group under $\otimes$. (If $\left\{\varrho_{\alpha \beta}\right\}$ and $\left\{\varrho_{\alpha \beta}^{\prime}\right\}$ are transition functions for $L \rightarrow M$ and $L^{\prime} \rightarrow M$ respectively, the tensor product $L \otimes L^{\prime} \rightarrow M$ has transition functions $\left.\left\{\varrho_{\alpha \beta} \cdot \varrho_{\alpha \beta}^{\prime}\right\}\right)$. However, the $n^{\text {th }}$ roots of $K$ 
do not form a subgroup of that group. They do form a coset of the subgroup of $n^{\text {th }}$ roots of the trivial bundle. If $U$ is an $n^{\text {th }}$ root of the trivial bundle, and $L$ is an $n^{\text {th }}$ root of $K$, then $L \otimes U$ is also an $n^{\text {th }}$ root. The degree of a line bundle over a compact Riemann surface $X$ is its Chern class $\left(\in H^{2}(X, \mathbb{Z}) \simeq \mathbb{Z}\right)$. If $X$ has genus $g$, the Chern class of $K(X)\left(\simeq T^{*}(X)\right)$ is $2 g-2$. If $L$ is an $n^{\text {th }}$ root of $K(X)$, and $L$ has degree $d$, then $(2 g-2)=\operatorname{deg} K(X)=\operatorname{deg}\left(L^{\otimes n}\right)=n d$. Thus $n^{\text {th }}$ roots of $K(X)$ exist only if $n \mid 2 g-2$.

Proposition 2.2. The canonical bundle of the universal Teichmüller curve is a holomorphic family of line bundles $K\left(V_{g}\right) \rightarrow V_{g} \rightarrow T_{g}$. The fibre over $t \in T_{g}$ is the canonical bundle $K\left(X_{t}\right)$.

Proof. The fibre over $t$ in $F_{g}=T_{g} \times \mathbb{C}$ is a set $D(t)$ homeomorphic to the unit disk, and $\Gamma_{g}$ acts on $F_{g}$ by the rule $\gamma(t, \zeta) \mapsto\left(t, \gamma^{t}(\zeta)\right)$, where for each $t \in T_{g}$ and $\gamma \in \Gamma_{g}, \gamma^{t}$ is a biholomorphic map of $D(t)$. This gives a convenient coordinate system for the quotient $V_{g}$, and the transition functions of $K\left(V_{g}\right)$ are

$$
\kappa_{\beta \alpha}=\operatorname{det}\left(D F_{\beta \alpha}^{-1}\right)^{t}=\left(\frac{\partial \gamma^{t}(\zeta)}{\partial \zeta}\right)^{-1},
$$

where $D F_{\beta \alpha}$ is the Jacobian matrix of the change of coordinates from $U_{\alpha}$ to $U_{\beta}$. For fixed $t, X_{t} \subseteq V_{g}$ is $D(t) /\left\{\gamma^{t} ; \gamma \in \Gamma_{g}\right\}$ and the right hand side of (2a) gives the transition functions for $K\left(X_{t}\right)$.

Over a single Riemann surface $X_{t}$, the canonical bundle is the cotangent bundle $T^{*}\left(X_{t}\right)$. Thus, $K\left(V_{g}\right)$ defines a holomorphic family of cotangent bundles over $T_{g}$. Similarly, the dual bundle defines a holomorphic family of tangent bundles $T\left(X_{t}\right)$ over $T_{g}$.

Corollary. Let $n$ be an integer dividing $2 g-2$ and $L \rightarrow V_{q}$ a holomorphic complex line bundle which is an $n^{\text {th }}$ root of $K\left(V_{g}\right) \rightarrow V_{g}$. Then $L \rightarrow V_{g} \rightarrow T_{g}$ is a holomorphic family of line bundles, and $L\left(X_{t}\right)$, the fibre over $t \in T_{g}$, is an $n^{\text {th }}$ root of $K\left(X_{t}\right)$ for each $t \in T_{g}$.

Remark. Clifford Earle made a preliminary study of $n^{\text {th }}$ roots using a different approach. In [4], he studied a fibre space $J\left(V_{g}\right) \rightarrow T_{g}$ such that each fibre over $t$ is the Jacobi variety $J\left(X_{t}\right)$. In [5], he constructed a fibre space $J^{\#}\left(V_{g}\right) \rightarrow T_{g}$ whose fibre over each point $t \in T_{g}$ is the group of divisor classes on the Riemann surface $X_{t}$. There is a canonical holomorphic section $\kappa: T_{g} \rightarrow J^{\#}\left(V_{g}\right)$ such that $\kappa(t)$ is the canonical divisor class on the Riemann surface $X_{t}$. He defines an $n^{\text {th }}$ root of $\kappa$ to be a holomorphic section $s: T_{g} \rightarrow J^{\#}\left(V_{g}\right)$ satisfying $n s=\kappa$ (here, $n$ is an integer dividing $2 g-2)$.

$\operatorname{Mod}\left(\Gamma_{g}\right)$ acts on these $n^{\text {th }}$ roots of $\kappa$. Moreover, $\hat{f} \in \operatorname{Mod}\left(\Gamma_{g}\right)$ in the kernel of that action implies that $\varrho(\hat{f})$ is congruent to $I(\bmod n)$ where $\varrho: \operatorname{Mod}\left(\Gamma_{g}\right) \rightarrow \operatorname{Sp}(g, \mathbb{Z})$ and $\operatorname{Sp}(g, \mathbb{Z})$ is the symplectic modular group. Earle proved that the condition is sufficient if $n=2$, and gave an example of $\hat{g} \in \operatorname{Mod}\left(\Gamma_{g}\right)$ with $\varrho(\hat{g})=I$ but $g$ does not act trivially on all the $(2 g-2)^{\text {th }}$ roots of $\kappa$. (This example is studied using geometric methods in Sect. 4.)

The relation between the approach in [5] and that used here is suggested by the classical correspondence (e.g. see [8] or [9]) between divisor classes and holomorphic complex line bundles over a Riemann surface. 
2.3. For any line bundle $\pi: L \rightarrow B$, let $\pi_{0}: L_{0} \rightarrow B$ be the fibre bundle obtained by removing the zero section from $L$. That is, $\pi_{0}^{-1}(x)=\mathbb{C}^{*}$, for any $x \in B$. If $F: L \rightarrow L^{\prime}$ is a bundle equivalence, then $F$ takes the zero section of $L$ to the zero section of $L^{\prime}$. We will denote the restriction to the punctured line bundle also by $F: L_{0} \rightarrow L_{0}^{\prime}$.

Lemma. If $L \rightarrow V_{B} \rightarrow T_{g}$ is a holomorphic family of line bundles, and $F: L \rightarrow L$ is a holomorphic bundle equivalence, then $F$ is given by multiplication by the complex number $f(t)$ in each fibre of $L \rightarrow T_{g}$, where $f$ is a holomorphic function of $t \in T_{g}$.

Proof. $F$ is fibre preserving and given by multiplication by a constant in each fibre of $L \rightarrow V_{g}$. The constant varies holomorphically as a function $f$ of $(t, \xi) \in V_{g}$. That function restricts to a holomorphic function on the compact Riemann surface $X_{t}$, where it must be a constant. Thus, $F(v)=f(t) \cdot v$ for all $v \in L_{0}$, where the holomorphic function $f$ depends only on $t$.

Corollary. If $F: L \rightarrow L$ is a holomorphic self-equivalence of the family $L \rightarrow V_{g} \rightarrow T_{g}$, then $F$ is homotopic to the identity.

Proof. Since $T_{g}$ is a simply connected region in $\mathbb{C}^{3 g-3}, \log (f(t))$ is a well-defined function in $T_{g}$. Then $H: I \times L \rightarrow L$ defined by $H(s, v)=e^{s \log f(t)} \cdot v$ is a homotopy between $F$ and the identity.

Proposition 2.3. If $L \rightarrow V_{g} \rightarrow T_{g}$ is an $n^{\text {th }}$ root of the canonical line bundle $K\left(V_{g}\right)$, then the equivalence $L^{\otimes n} \simeq K\left(V_{g}\right)$ induces a covering map $p: L_{0} \rightarrow K_{0}\left(V_{g}\right)$. Changing the equivalence produces another covering map $p^{\prime}$ with $p^{\prime}=f(t) p$, where $f$ is a holomorphic function on $T_{g}$.

Proof. One checks easily that the map given in local coordinates by $P\left(v_{\alpha}, \xi_{\alpha}\right)=\left(v_{\alpha}, \xi_{\alpha}^{n}\right)$ is a well defined map $P: L \rightarrow L^{\otimes n}$. Since $\mathbb{C}^{*} \rightarrow \mathbb{C}^{*}$ by $z \mapsto z^{n}$ is an $n$-fold covering map, $P: L_{0} \rightarrow L_{0}^{\otimes_{n}}$ is an $n$-fold covering. Because $L$ is an $n^{\text {th }}$ root, there is an equivalence $F: L^{\otimes n} \rightarrow K\left(V_{g}\right)$. The composition $p=F \circ P$ is the desired $n$-fold covering map. If $G$ is another equivalence, then $G \circ F^{-1}$ is a holomorphic self-equivalence of $K\left(V_{g}\right)$, and the second statement follows from the lemma.

2.4. We now want to define the action of $\operatorname{Mod}\left(\Gamma_{g}\right)$ on the $n^{\text {th }}$ roots of $K\left(V_{g}\right)$. We fix a standard system of generators for $\Gamma_{g}$ (hence also for $H_{1}(S, \mathbb{Z})$ ), that is, a set $\left\{A_{1}\right.$, $\left.A_{2}, \ldots, A_{g}, B_{1}, B_{2}, \ldots, B_{g}\right\}$ of generators which satisfy the single defining relation $\prod_{j=1}^{g} A_{j} B_{j} A_{j}^{-1} B_{j}^{-1}=1$ and so that $\left(A_{j} \times A_{k}\right)=\left(B_{j} \times B_{k}\right)=0$ and $\left(A_{j} \times B_{k}\right)=\delta_{j k}, 1 \leqq j$, $k \leqq g$. Here $(\gamma \times \beta)$ means the algebraic intersection number of the closed loops $\gamma$ and $\beta$ on $S$.

Every diffeomorphism $f: S \rightarrow S$ induces an automorphism of $\pi_{1}(S, x)=\Gamma_{g}$. An automorphism $\alpha$ of $\Gamma_{g}$ induces a unique automorphism of $H_{1}(S, \mathbb{Z})$; let $\varrho(\alpha)$ be the matrix of that automorphism with respect to the homology basis which has been fixed. The automorphism $\varrho(\alpha)$ preserves the intersection matrix of the homology basis if and only if $\alpha$ is included by a sense preserving diffeomorphism, or equivalently,

$$
[\varrho(\alpha)]^{t} J[\varrho(\alpha)]=J \text {. }
$$

Here $J=\left[\begin{array}{cc}0 & -I \\ I & 0\end{array}\right]$ and $N^{t}$ is the transpose of the matrix $N$. 
The group of all automorphisms of $\Gamma_{g}$ satisfying (2b) is denoted by $\bmod \left(\Gamma_{g}\right)$. Associating each $\gamma_{0}$ in $\Gamma_{g}$ to the automorphism $\gamma \mapsto \gamma_{0} \gamma \gamma_{0}^{-1}$ identifies $\Gamma_{g}$ with the normal subgroup of inner automorphisms. The Teichmüller modular group $\operatorname{Mod}\left(\Gamma_{q}\right)$ is the quotient group $\bmod \left(\Gamma_{q}\right) / \Gamma_{g}$. Denote the group of sense preserving diffeomorphisms of $S$ by $\operatorname{Diff}^{+}(S)$ and the normal subgroup of those homotopic to the identity by $\operatorname{Diff}_{0}(S)$. The mapping class group of $S$ is by definition $\operatorname{Diff}^{+}(S) / \operatorname{Diff}_{0}(S)$; it is well known (e.g. see [13] for a discussion) that this group can be identified with $\operatorname{Mod}\left(\Gamma_{g}\right)$. If $f$ is an element of $\operatorname{Diff}^{+}(S)\left[\bmod \bmod \left(\Gamma_{g}\right)\right]$ we denote its equivalence class in $\operatorname{Mod}\left(\Gamma_{g}\right)$ by $\hat{f}$.

The symplectic modular group $\operatorname{Sp}(g, \mathbb{Z})$ is the group of $2 g \times 2 g$ integer matrices satisfying (2b). The mapping $\varrho$ which takes an element of $\operatorname{Mod}\left(\Gamma_{g}\right)$ to the matrix representing its action on homology is a homomorphism; $\varrho$ maps onto $\operatorname{Sp}(g, \mathbb{Z})$ [17].

According to Bers [2], $\bmod \left(\Gamma_{q}\right)$ and $\operatorname{Mod}\left(\Gamma_{g}\right)$ act as groups of biholomorphic mappings on $F_{g}$ and $T_{g}$ respectively. We will need the following important properties of these actions:

1. $t, s \in T_{g}$ represent conformally equivalent Riemann surfaces if and only if there is a $\hat{g} \in \operatorname{Mod}\left(\Gamma_{g}\right)$ with $s=\hat{g}(t)$.

2. $\operatorname{Mod}\left(\Gamma_{g}\right)$ acts on $V_{g}$ as a group of biholomorphic maps; $f \in \operatorname{Diff}^{+}(S)$ induces $\hat{f}: V_{g} \rightarrow V_{g} . X_{t}=\pi^{-1}(t)$ and $X_{\hat{f}(t)}=\pi^{-1}(\hat{f}(t))$ are conformally equivalent Riemann surfaces. Indeed, $\hat{f}: V_{g} \rightarrow V_{g}$ restricts to a conformal equivalence $\hat{f}_{t}: X_{t} \rightarrow X_{\hat{f}(t)}$.

3. In view of Proposition 2.1, $\operatorname{Mod}\left(\Gamma_{g}\right)$ also acts as a group of sense-preserving diffeomorphisms on $T_{g} \times S$, and the sense preserving diffeomorphism $\theta^{t} \hat{f}_{t}\left(\theta^{t}\right)^{-1}$ : $S \rightarrow S$ is isotopic to $f$.

The biholomorphic mapping $\hat{f}: V_{g} \rightarrow V_{g}$ induces maps on the canonical bundle and its dual as follows:

Definition. a) $\hat{f}: V_{g} \rightarrow V_{g}$ inducies a mapping $K^{*}(\hat{f})$ on $K^{*}\left(V_{g}\right)$ which is given in local coordinates by

$$
K^{*}(\hat{f})(v, \xi)=(\hat{f}(v), \operatorname{det} D \hat{f} \cdot \xi),
$$

where $D \hat{f}$ is the Jacobian matrix in appropriate local coordinates. Here, we can think of $\xi \in \mathbb{C}$ as representing $\xi \cdot \frac{\partial}{\partial t_{1}} \wedge \ldots \wedge \frac{\partial}{\partial t_{m-1}} \wedge \frac{\partial}{\partial \zeta}$, where $m=3 g-2=\operatorname{dim} V_{g}$.

b) Similarly, on $K\left(V_{g}\right)$,

$$
K(\hat{f})(v, \eta)=\left(\hat{f}(v), \operatorname{det}\left(D \hat{f}^{-1}\right)^{t} \cdot \eta\right),
$$

where we can think of $\eta \in \mathbb{C}$ as representing $\eta d t_{1} \wedge \ldots \wedge d t_{m-1} \wedge d \zeta$.

Remark. For fixed $t \in T_{g}, \hat{f}_{t}: X_{t} \rightarrow X_{\hat{f}(t)}$ is a biholomorphism, so there is a differential $T\left(\hat{f}_{t}\right): T\left(X_{t}\right) \rightarrow T\left(X_{\hat{f}(t)}\right)$ and a "codifferential" $T^{*}\left(\hat{f}_{t}\right): T^{*}\left(X_{t}\right) \rightarrow T^{*}\left(X_{\hat{f}(t)}\right)$ on the tangent and cotangent bundles. The maps $K \hat{f}_{t}$ and $K^{*}\left(\hat{f}_{t}\right)$ are defined so that their restrictions

and

$$
K^{*} \hat{f}_{t}: K^{*}\left(X_{t}\right) \rightarrow K^{*}\left(X_{\hat{f}(t)}\right)
$$

$$
K \hat{f}_{t}: K\left(X_{t}\right) \rightarrow K\left(X_{f(t)}\right)
$$


are the differential and the codifferential under the identification of $K^{*}\left(X_{t}\right)$ and $K\left(X_{t}\right)$ with the tangent and cotangent bundles, respectively.

Proposition 2.4. Let $L$ be an $n^{\text {th }}$ root of $K\left(V_{g}\right)$. Then the diagram

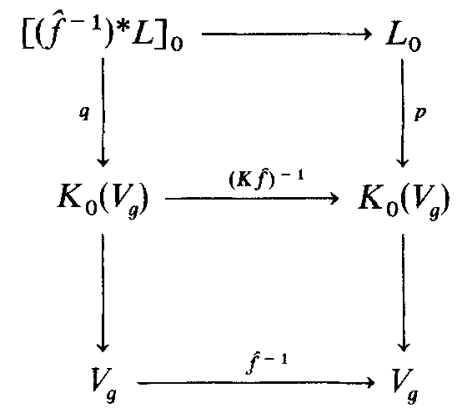

commutes, where $\left(\hat{f}^{-1}\right)^{*} L$ is the pullback.

Proof. First we notice that the pullback $\left(\hat{f}^{-1}\right)^{*}\left(K\left(V_{g}\right)\right)$ is equivalent to $K\left(V_{g}\right)$, because the diagram

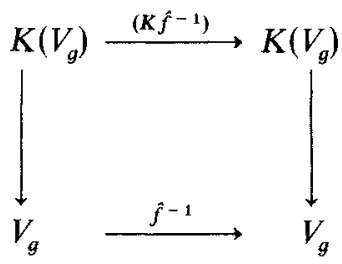

commutes (using a uniqueness theorem for pullbacks).

We have a mapping $p: L \rightarrow K\left(V_{g}\right)$ which is a covering mapping on the punctured line bundles (as in Proposition 2.3). Thus, we get the diagram as claimed by taking pullbacks of $K\left(V_{g}\right)$ and $L$ by the mapping $\hat{f}^{-1}$.

Corollary. The action of $\operatorname{Mod}\left(\Gamma_{g}\right)$ on $V_{g}$ induces a left action on the set of $n^{\text {th }}$ roots of $K\left(V_{g}\right)$. If $\hat{f} \in \operatorname{Mod}\left(\Gamma_{g}\right)$, we write

where $L$ is an $n^{\text {th }}$ root.

$$
\hat{f} \cdot L=\left(\hat{f}^{-1}\right)^{*} L,
$$

Proof. By comparing transition functions, we see that $L^{\otimes n} \simeq K\left(V_{g}\right)$ implies that $\left[\left(\hat{f}^{-1}\right)^{*}(L)\right]^{\otimes n} \simeq\left(\hat{f}^{-1}\right)^{*}\left(K\left(V_{g}\right)\right)\left(\simeq K\left(V_{g}\right)\right)$. Thus $\left(\hat{f}^{-1}\right)^{*}(L)$ is an $n^{\text {th }}$ root of $K\left(V_{g}\right)$, and the map $q$ is a covering map on the punctured bundles.

2.5. Our next goal is to describe a certain set of homomorphisms $H_{1}\left(T_{0}(S)\right.$, $\left.\mathbb{Z}_{n}\right) \rightarrow \mathbb{Z}_{n}$. We will see in the next section that $\operatorname{Mod}\left(\Gamma_{g}\right)$ acts on this set.

The loop around the origin in a punctured holomorphic complex line bundle $L_{0} \rightarrow B$ ( $B$ is a complex manifold) is the homology class of a closed path $\gamma$ oriented counter-clockwise around a circle centered at zero in any fibre $\mathbb{C}^{*}$ of $L_{0}$. It is easy to see that the homology class of $\gamma$ is well-defined and preserved by complex bundle equivalences. An equivalence of the underlying real bundles may reverse the orientation. 
We continue with our fixed smooth surface $S$ and its preferred Riemann surface structure $X_{0}$. Let $\alpha$ be the loop around the origin in the punctured tangent bundle $T_{0}\left(X_{0}\right)$. "Forgetting the complex structure" on $T_{0}\left(X_{0}\right)$, we regard $\alpha$ as belonging to the homology group $H_{1}\left(T_{0}(S), \mathbb{Z}_{n}\right)$.

Definition. Let $\alpha$ be the loop around the origin in $T_{0}(S)$, as above. We define

$$
\Lambda\left(T_{0}(S)\right)=\left\{\text { homomorphisms } \lambda: H_{1}\left(T_{0}(S), \mathbb{Z}_{n}\right) \rightarrow \mathbb{Z}_{n} \text { such that } \lambda(\alpha)=-1\right\} \text {. }
$$

$\Lambda\left(T_{0}(S)\right)$ is nonempty if and only if $n$ divides the order of $\alpha$, which we find in the next proposition.

Proposition 2.5. $H_{1}\left(T_{0}(S), \mathbb{Z}\right) \simeq \mathbb{Z}^{2 g} \times \mathbb{Z}_{2 g-2}$. Here, $\mathbb{Z}_{2 g-2}$ is generated by $\alpha$, the loop around the origin in $T_{0}(S)$. If $\left\{A_{i}, B_{i}\right\} \quad(i=1,2, \ldots, g)$ is a standard system of generators for $H_{1}(S, \mathbb{Z})$, we choose lifts $\left\{\tilde{A}_{i}, \tilde{B}_{i}\right\} \in H_{1}\left(T_{0}(S), \mathbb{Z}\right)$. Then $\left\{\tilde{A}_{i}, \tilde{B}_{i}\right\}$ generate $\mathbb{Z}^{2 g}$.

Proof. Since $H_{2}(S) \simeq \mathbb{Z}, H_{0}(S) \simeq \mathbb{Z}$ and $H_{1}(S) \simeq \mathbb{Z}^{2 g}$, the Gysin sequence for the tangent bundle ends with

$$
\rightarrow \mathbb{Z} \rightarrow \mathbb{Z} \rightarrow H_{1}\left(T_{0}(S), \mathbb{Z}\right) \rightarrow \mathbb{Z}^{2 g} \rightarrow 0 .
$$

That exact sequence has the following two properties:

a) The map $\mathbb{Z} \rightarrow H_{1}\left(T_{0}(S), \mathbb{Z}\right)$ takes the generator of $\mathbb{Z}$ to the loop around the origin in $T_{0}(S)$.

b) The map $\mathbb{Z} \rightarrow \mathbb{Z}$ is multiplication by $( \pm)(2 g-2)$. Therefore, we get the short exact sequence

$$
0 \rightarrow \mathbb{Z}_{2 g-2} \rightarrow H_{1}\left(T_{0}(S), \mathbb{Z}\right) \rightarrow H_{1}(S, \mathbb{Z}) \rightarrow 0 .
$$

Because of a), $\alpha$ generates $\mathbb{Z}_{2 g-2}$, and the generators of $\mathbb{Z}^{2 g}$ are inverse images of a basis for $H_{1}(S, \mathbb{Z})$ under the map $H_{1}\left(T_{0}(S), \mathbb{Z}\right) \rightarrow H_{1}(S, \mathbb{Z})$.

Remark. Of course, these lifts $\left\{\widetilde{A_{i}}, \widetilde{B_{i}}\right\}$ are not unique; two lifts of the same curve can differ by a multiple of the loop around the origin. We fix a particular set of generators for $H_{1}(S, \mathbb{Z})$ consisting of regular simple closed curves $\left\{A_{1}, A_{2}, \ldots, A_{g}\right.$, $\left.B_{1}, B_{2}, \ldots, B_{g}\right\}$ parameterized so that their tangent vectors have length 1 . Moreover, we think of $S$ as having its $g$ handles arranged symmetrically around the surface and require that the rotation of order $g$ takes $A_{i} \rightarrow A_{i+1}$ and $B_{i} \rightarrow B_{i+1}$ for $i \neq g$, and $A_{g}, B_{g} \rightarrow A_{1}$ and $B_{1}$, respectively. This system of generators is shown in Fig. 1.

For any regular curve $\gamma$ in $S$, by $\bar{\gamma}$ we mean the particular lift of $\gamma$ to $T_{0}(S)$ which consists of $\gamma$ together with its tangent vector at every point, i.e.

$$
\vec{\gamma}(t)=\left(\gamma(t), \gamma^{\prime}(t)\right) \text {. }
$$

The basis we have chosen for $H_{1}(S, \mathbb{Z})$ thus determines the basis $\left\{\bar{A}_{1}, \ldots, \bar{A}_{g}\right.$, $\left.\bar{B}_{1}, \ldots, \bar{B}_{g}, \alpha\right\}$ for $H_{1}\left(T_{0}(S) ; \mathbb{Z}_{n}\right)=H_{1}\left(K_{0}^{*}\left(X_{0}\right), \mathbb{Z}_{n}\right)$. These bases should be regarded as fixed for the remainder of the paper.

Corollary. If $n$ divides $2 g-2, H_{1}\left(T_{0}(S), \mathbb{Z}_{n}\right) \simeq\left(\mathbb{Z}_{n}\right)^{2 g+1}$.

The set $A\left(T_{0}(S)\right)$ is nonempty if and only if $n$ divides $2 g-2$. In this case, the elements of $A\left(T_{0}(S)\right)$ can be represented with respect to our fixed basis as row 


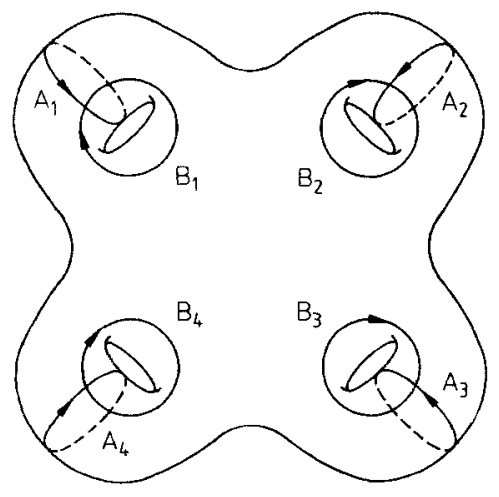

Fig. 1

vectors in $\left(\mathbb{Z}_{n}\right)^{2 g+1}$ with final entry -1 . Thus $\Lambda\left(T_{0}(S)\right)$ is a finite set of order $n^{2 g}$. Also, each of these vectors specifies a homomorphism from $H_{1}\left(S, \mathbb{Z}_{n}\right) \rightarrow \mathbb{Z}_{n}$, that is, an element of $H^{1}\left(S, \mathbb{Z}_{n}\right)$. Thus, $\Lambda\left(T_{0}(S)\right)$ is in $1-1$ correspondence with $H^{1}\left(S, \mathbb{Z}_{n}\right)$, although this correspondence is not natural.

Remark. $H_{1}\left(T_{0}(S), \mathbb{Z}_{n}\right)$ can be computed in another way. It follows from Massey's appendix in [1] (see also pp. 37-41 and 23-25 of [1]) that $\pi_{1}\left(T_{0}(S)\right.$ ) is the group with generators $\left\{\widetilde{A_{i}}, \widetilde{B_{i}}, \alpha\right\}(i=1,2, \ldots, g)$ and relations

$$
\begin{gathered}
\alpha^{2 g-2} \prod_{i=1}^{g}\left[\widetilde{A_{i}}, \widetilde{B_{i}}\right]=1 \\
\widetilde{A_{i}} \alpha=\alpha \widetilde{A_{i}} \\
\widetilde{B_{i}} \alpha=\alpha \widetilde{B_{i}} .
\end{gathered}
$$

Abelianizing this group gives the result of Proposition 2.5.

2.6. We can now define the action of $\operatorname{Mod}\left(\Gamma_{g}\right)$ on $\Lambda\left(T_{0}(S)\right)$.

Proposition 2.6. $\operatorname{Mod}\left(\Gamma_{g}\right)$ acts on $\Lambda\left(T_{0}(S)\right)$ by the rule

$$
\hat{f} \cdot \lambda=\lambda \circ\left(T f^{-1}\right)_{*} \text { for all } \hat{f} \in \operatorname{Mod}\left(\Gamma_{g}\right) .
$$

Proof. If $f_{1}$ and $f_{2}$ are isotopic $\left(\hat{f}_{1}=\hat{f}_{2}\right)$, one checks that $\left(T f_{1}\right)$ and $\left(T f_{2}\right)$ are also isotopic, and therefore induce the same map on homology. (The idea is that the differential of the isotopy between $f_{1}$ and $f_{2}$ gives an isotopy between $T f_{1}$ and $T f_{2}$.) Also, $f$ is sense-preserving, so the differential $T f^{-1}$ takes the loop around the origin to itself. Hence $\hat{f} \cdot \lambda(\alpha)=-1$, and we see that $\hat{f} \cdot \lambda$ is a well-defined element of $\Lambda\left(T_{0}(S)\right)$. Since

$$
(\hat{f} \circ \hat{g}) \cdot \lambda=\lambda \circ T(f \circ g)_{*}^{-1}=\lambda \circ\left(T_{g}^{-1}\right)_{*} \circ\left(T f^{-1}\right)_{*}=\hat{f} \cdot\left(\lambda \circ\left(T_{g}^{-1}\right)_{*}\right)=\hat{f} \cdot \hat{g} \cdot \lambda,
$$

the rule (2f) gives a left action of $\operatorname{Mod}\left(\Gamma_{g}\right)$ on $A\left(T_{0}(S)\right)$.

Note that $\left(T f^{-1}\right)_{*}$ can be expressed as a $(2 g+1) \times(2 g+1)$ matrix $(\bmod n)$ with respect to the basis for $H_{1}\left(T_{0}(S), \mathbb{Z}_{n}\right)$ chosen in Sect. 2.5. Having used the same basis to represent $\Lambda\left(T_{0}(S)\right)$ as a set of row vectors, we can compute the right hand side of formula (2f) by matrix multiplication. 
2.7. We outline the proof of

Theorem A. The subgroup $G_{g, n}$ of $\operatorname{Mod}\left(\Gamma_{g}\right)$ which acts trivially on all the $n^{\text {th }}$ roots is precisely the subgroup of elements which induce the identity $(\bmod n)$ on the homology of the unit tangent bundle of $S$.

It is easy to see from the matrix description of the action of $\operatorname{Mod}\left(\Gamma_{g}\right)$ on $A\left(T_{0}(S)\right)$ that $\hat{f} \in \operatorname{Mod}\left(\Gamma_{g}\right)$ acts trivially on all elements of $\Lambda\left(T_{0}(S)\right)$ if and only if $\left(T f^{-1}\right)_{*} \equiv I(\bmod n)$. Thus Theorem A follows readily from the theorem:

Theorem 1. Let $L \rightarrow K\left(V_{g}\right) \rightarrow V_{g}$ be an $n^{\text {th }}$ root of $K\left(V_{g}\right)$. The covering map $p: L_{0} \rightarrow K_{0}\left(V_{g}\right)$ determines a homomorphism in $\Lambda\left(T_{0}(S)\right)$. That correspondence induces an equivariant bijection between the set of (equivalence classes of) $n^{\text {th }}$ roots of the canonical bundle and the set $\Lambda\left(T_{0}(S)\right)$.

Theorem 1 will be broken up into several parts and proved in Sects. 5 and 6 .

\section{Computing the Action of $\operatorname{Mod}\left(\Gamma_{g}\right)$ on Generators (Theorem B)}

Viewing the $n^{\text {th }}$ roots as homomorphisms in $A\left(T_{0}(S)\right)$, and the action of $\operatorname{Mod}\left(\Gamma_{g}\right)$ as in (2f) has the advantage that the action can be computed on a set of generators for $\operatorname{Mod}\left(\Gamma_{g}\right)$. We begin by fixing a (finite) set of generators which is useful for our computations.

Let $\gamma$ be a simple closed curve on $S$. Let $j$ be an orientation-preserving embedding of the cylinder $[-1,1] \times S^{1}$ into $S$ with $j\left(\{0\} \times S^{1}\right)=\gamma$. The (positive) Dehn twist $f_{\gamma}$ about the loop $\gamma$ is the homeomorphism of $S$ onto itself defined by

$$
\begin{aligned}
f_{y}(x) & =x \quad \text { if } \quad x \notin \operatorname{Im}(j) . \\
f_{y}(j(t, \theta)) & =j(t, \theta+(t+1) \pi) .
\end{aligned}
$$

This homeomorphism is homotopic to a sense-preserving diffeomorphism of $S$ which we will also denote by $f_{\gamma}$. The element $\hat{f}_{\gamma}$ of $\operatorname{Mod}\left(\Gamma_{g}\right)$ so determined is independent of the embedding $j$ and independent of the orientation of the loop $\gamma$. The effect of the Dehn twist $\hat{f}_{y}$ on the homology of the surface $S$ is given by the formula

$$
\left(\hat{f}_{\gamma}\right)_{*}(\beta)=\beta+(\gamma \times \beta) \cdot \gamma \quad \forall \beta \in H_{1}(S, \mathbb{Z})
$$

Lickorish $[11,12]$ proved that the twists about the loops in Fig. 2a generate $\operatorname{Mod}\left(\Gamma_{g}\right)$. Therefore, the Dehn twists $f_{A_{1}}, f_{B_{1}}, f_{C_{1}}$ about the three loops $A_{1}, B_{1}, C_{1}$ shown (in the case $g=4$ ) in Fig. 2b together with $r_{g}$, the rotation of order $g$, generate $\operatorname{Mod}\left(\Gamma_{g}\right)$ (see Mumford [16]).

Notation. We denote curves in $T_{0}(S)$ by $\tilde{\tau}$, their images under $\pi: T_{0}(S) \rightarrow S$ by $\tau$. For any loop $\gamma$ (or $\tilde{\gamma}$ ), we denote the homology class by $[\gamma]$ (or $[\tilde{\gamma}]$ ).

Lemma. Let $f$ be any surface diffeomorphism, $\gamma$ any regular curve on $S$ and $\vec{\gamma}$ its lift as in Eq. (2e). Then the induced map on homology $\left(H_{1}\left(T_{0}(S), \mathbb{Z}_{n}\right)\right)$ is given by the formula

$$
(T f)_{*}([\bar{\gamma}])=[\overline{f(\gamma)}] .
$$

Proof. $(T f)_{*}(\bar{\gamma}(t))=T f\left(\gamma(t), \gamma^{\prime}(t)\right)=\left(f(\gamma(t)), \frac{\partial}{\partial t}(f \circ \gamma)\right)=(\overline{f \circ \gamma}(t))$ by the chain rule. 


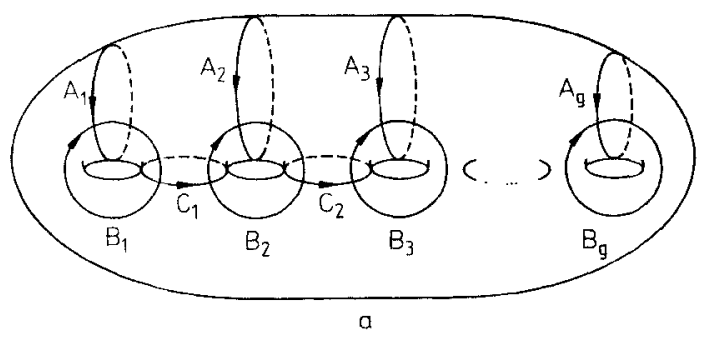

Fig. $2 a$ and $b$

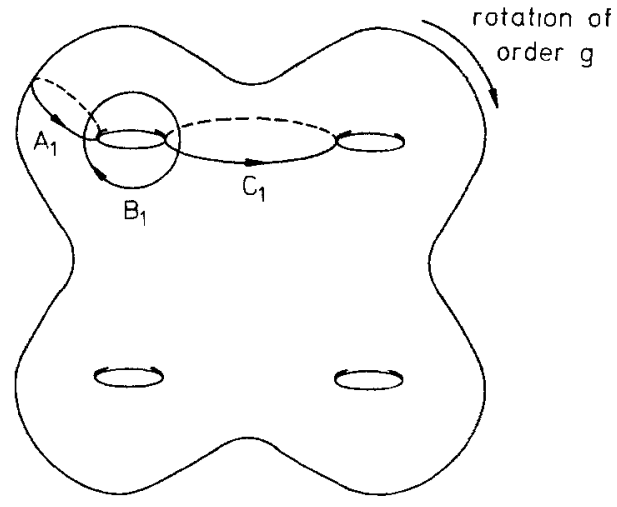

b

Proposition 3.1. Let $\gamma$ be a regular curve on $S, f_{\gamma}$ the Dehn twist about $\gamma$. Then action of $f_{\gamma} \in \operatorname{Mod}\left(\Gamma_{g}\right)$ on $H_{1}\left(T_{0}(S), \mathbb{Z}_{n}\right)$ is then given by the formula

$$
\left(T f_{\gamma}\right)_{*}(\tilde{\tau})=\tilde{\tau}+(\gamma \times \tau)[\bar{\gamma}],
$$

where $\tilde{\tau}$ is any element in $H_{1}\left(T_{0}(S), \mathbb{Z}_{n}\right)$.

Proof. Recall that $(T f)_{*}(\alpha)=\alpha$ for any sense-preserving diffeomorphism $f$. We first prove that (3c) holds for $\gamma=B_{1}$. Of course, for any $\tilde{\tau}$ with $(\gamma \times \tau)=0$, the induced map on homology leaves $\tilde{\tau}$ fixed. To compute $\left(T f_{B_{1}}\right)_{*}\left[\bar{A}_{1}\right]$, notice that $\left(B_{1} \times A_{1}\right)=-1$. Then $\left(T f_{B_{1}}\right)_{*}\left[\bar{A}_{1}\right]$, by the lemma, is the homology class of the curve in Fig. 3a, with its tangent vector as indicated. But that curve is homotopic in $T_{0}(S)$ to the curve in Fig. $3 \mathrm{~b}$ where the tangent vectors at the one point of intersection coincide. The curve can then be written as the sum of two closed loops in $T_{0}(S)$, as in Fig. 3c. Therefore $\left(T f_{B_{1}}\right)_{*}\left(\left[\bar{A}_{1}\right]\right)=\left[\bar{A}_{1}\right]+\left(B_{1} \times A_{1}\right)\left[\bar{B}_{1}\right]$. That proves the formula for all elements of the basis $\left\{\bar{A}_{1}, \ldots, \bar{B}_{g}, \alpha\right\}$, since $\left(B_{1} \times \tau\right)=0$ for all other $\tilde{\tau}$ in the basis. The formula $\left(T f_{B_{1}}\right)_{*}(\tilde{\tau})=\tilde{\tau}+\left(B_{1} \times \tau\right)\left[\bar{B}_{1}\right]$ follows easily for arbitrary $\tilde{\tau} \in H_{1}\left(T_{0}(S), \mathbb{Z}_{n}\right)$, by expressing $\tilde{\tau}$ in terms of the basis elements. So the proposition is true for $\gamma=B_{1}$.

Now suppose that $\gamma$ is any regular (closed) curve on $S$. If $\gamma$ is a dividing cycle, then $[\gamma]=0$, and its intersection numbers with the standard basis are all zero, so the formula holds. If $\gamma$ is not a dividing cycle, then there is a standard basis so that $\gamma=B_{1}$, and the above argument shows the formula holds.

Notation. Recall that $\varrho$ is the homomorphism from $\operatorname{Mod}\left(\Gamma_{n}\right)$ to the symplectic group $\operatorname{Sp}(g, \mathbb{Z}) . \varrho(f)$ is a $2 g \times 2 g$ matrix $(\bmod n)$ which describes the action of the surface diffeomorphism on the homology of $S$. Let $R=\left(r_{i j}\right), E=\left(e_{i j}\right), M=\left(m_{i j}\right)$ and $I$ be the $g \times g$ matrices

$I=$ identity

$R=$ the permutation matrix $r_{i j}=\delta_{i, j+1}, 1 \leqq j \leqq g-1$

$r_{i g}=\delta_{i, 1}, i=g$.

$E=$ matrix with $e_{11}=1$, all other entries 0 .

$M=\left(m_{i j}\right)$, where $m_{11}=m_{22}=1, m_{12}=m_{21}=-1$, all other entries 0 . 

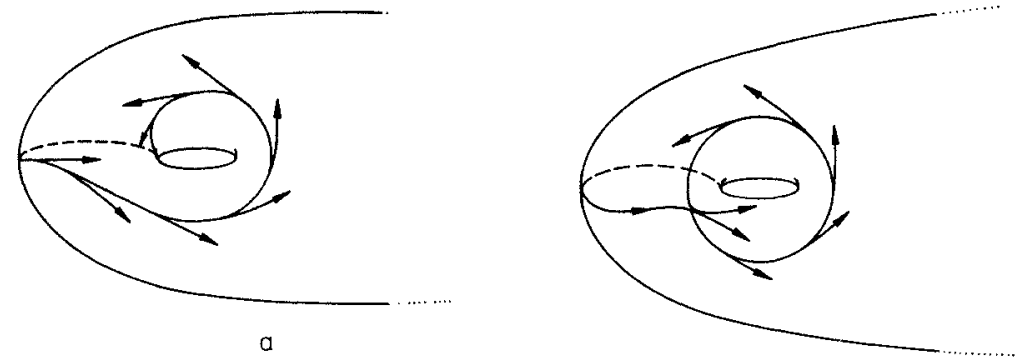

$b$

Fig. 3a-c
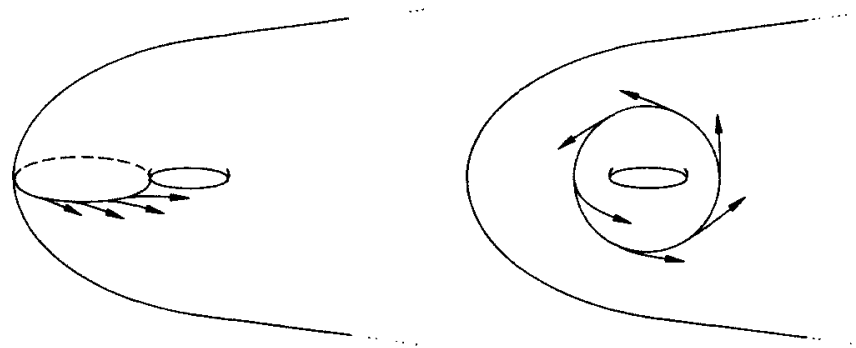

$c$

One can verify by direct computation [Eq. (3a) simplifies the first three] that the generators $f_{A_{1}}, f_{B_{1}}, f_{C_{1}}, r_{g}$ act on the homology of the surface $S$ by

$$
\begin{array}{ll}
\varrho\left(f_{A_{1}}\right)=\left(\begin{array}{ll}
I & E \\
0 & I
\end{array}\right) & \varrho\left(f_{B_{1}}\right)=\left(\begin{array}{rr}
I & 0 \\
-E & I
\end{array}\right) \\
\varrho\left(f_{C_{1}}\right)=\left(\begin{array}{cc}
I & M \\
0 & I
\end{array}\right) & \varrho\left(r_{g}\right)=\left(\begin{array}{ll}
R & 0 \\
0 & R
\end{array}\right) .
\end{array}
$$

This is also obvious from the proof of the next theorem.

We can think of the loop $\alpha$ around the origin in the fibre of $T_{0}(S)$ as the lift [in the sense of (2e)] of a small loop on $S$, homotopic to a point, traversed in the counter-clockwise direction. Here, a "small" loop is one which is contained in a single coordinate patch.

In the following arguments, we will specify a loop in $T_{0}(S)$ by drawing a loop on the surface, and indicating a choice of tangent vector (tangent to the surface, not necessarily to the curve) at each point on the path. Thus, the arrows in the drawings (except in Figs. $4 a$ and 5a) indicate the location of the point in the fibre of $T_{0}(S)$ and not the direction in which the path is traversed. That will be determined by the boundary orientation, unless explicitly specified otherwise.

Proposition 3.2. The maps induced on the homology of $T_{0}(S)$ by $r_{g}, f_{A_{1}}, f_{B_{1}}$, and $f_{C_{1}}$ are represented (with respect to our basis $\left\{\bar{A}_{1}, \bar{A}_{2}, \ldots, \bar{A}_{g}, \bar{B}_{1}, \ldots, \bar{B}_{g}, \alpha\right\}$ ) by the 

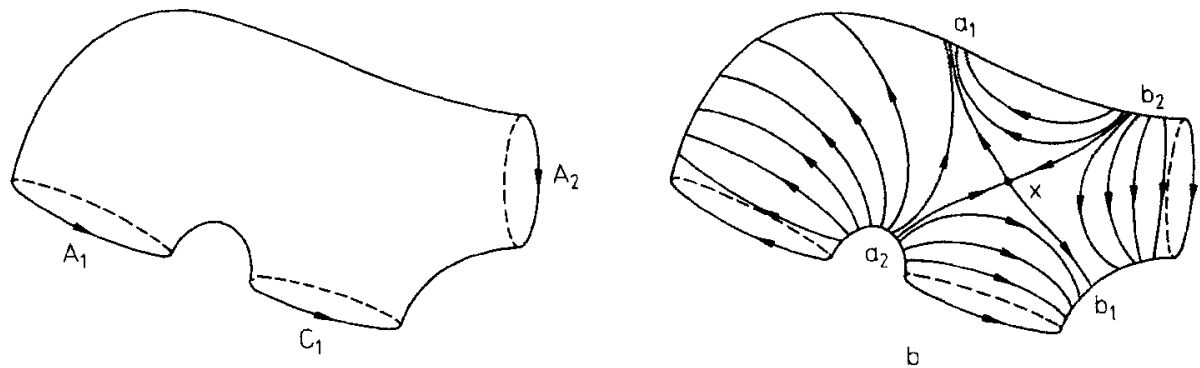

a

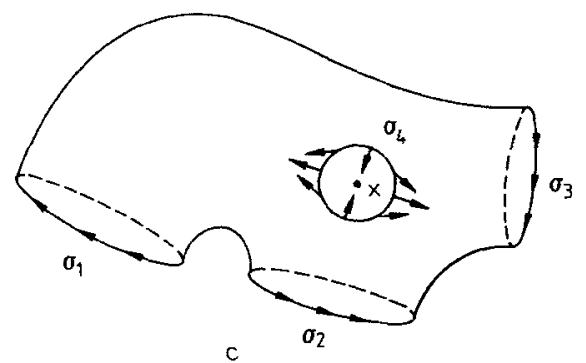

Fig. 4a-c

$(2 g+1) \times(2 g+1)$ matrices

$$
\begin{array}{ll}
\left(T r_{g}\right)_{*}=\left(\begin{array}{cc}
\varrho\left(r_{g}\right) & 0 \\
0 & 1
\end{array}\right) & \left(T f_{A_{1}}\right)_{*}=\left(\begin{array}{cc}
\varrho\left(f_{A_{1}}\right) & 0 \\
0 & 1
\end{array}\right) \\
\left(T f_{B_{1}}\right)_{*}=\left(\begin{array}{cc}
\varrho\left(f_{B_{1}}\right) & 0 \\
0 & 1
\end{array}\right) & \left(T f_{C_{1}}\right)_{*}=\left(\begin{array}{ccc}
I & M & 0 \\
0 & I & 0 \\
0 & v & 1
\end{array}\right),
\end{array}
$$

where $v=\left(v_{1}, \ldots, v_{g}\right)$ is the row vector with $v_{1}=1, v_{2}=-1, v_{i}=0$ for $3 \leqq i \leqq g$.

Proof. For $r_{g}$ : Since $r_{g}$ takes $A_{1}$ (with its tangent vectors) to $A_{2}$ (with its tangent vectors) and the loop around the origin is left fixed, the matrix for $\left(\operatorname{Tr}_{g}\right)_{*}$ is as claimed. For $f_{A_{1}}$ and $f_{B_{1}}$ : For any element $\tilde{\tau}$ in the basis, $\left(A_{1} \times \tau\right)=0$ unless $\tilde{\tau}=\bar{B}_{1}$, in which case $\left(A_{1} \times B_{1}\right)=1$. From (3c) we conclude $\left(T f_{A_{1}}\right)_{*}\left(\bar{B}_{1}\right)=\bar{B}_{1}+\bar{A}_{1}$, but $\left(T f_{A_{1}}\right)_{*}$ fixes every other basis element. Similarly, $\left(T f_{B_{1}}\right)_{*}$ fixes every basis element except $\bar{A}_{1}$, and $\left(T f_{B_{1}}\right)_{*}\left(\bar{A}_{1}\right)=\bar{A}_{1}-\bar{B}_{1}$, so we get the matrices for $\left(T f_{A_{1}}\right)_{*}$ and $\left(T f_{B_{1}}\right)_{*}$ as claimed. For $f_{C_{1}}$ : Our first step in computing this action is to compute $\bar{C}_{1}$ in terms of the basis vectors.

We look at the portion of the surface bounded by $A_{1}, C_{1}$ and $A_{2}$ as indicated in Fig. 4a. (The arrows there indicate the orientation of the paths $A_{1}, A_{2}, C_{1}$.) Take the usual orientation on the surface. The boundary of this piece of surface is, of course, homologous $(\sim)$ to zero, which says

$$
A_{1}+C_{1}-A_{2} \sim 0 \text {. }
$$

The idea now is to lift the surface of Fig. 4 a to a surface in $T_{0}(S)$. We do this by drawing a vector field on the surface, with its only singularity at the point $x$. Such a vector field is illustrated in Fig. $4 b$ (the extension to the back of the surface which 
is not visible is non-singular; flow lines on the back of the surface connect $a_{1}$ to $a_{2}$ and $b_{1}$ to $b_{2}$ ). Take a small neighborhood around $x$, bounded by a loop homotopic to the point $x$, and contained in a single coordinate neighborhood of $T_{0}(S)$. If we remove this neighborhood from the surface, we get a smooth vector field. We see that the four closed loops $\sigma_{1}, \sigma_{2}, \sigma_{3}, \sigma_{4}$ in $H_{1}\left(T_{0}(S), \mathbb{Z}_{n}\right)$ indicated in Fig. $4 \mathrm{c}$ bound a surface in $T_{0}(S)$. The arrows are tangent vectors, the restriction of the above vector field to the boundary curves. With the induced boundary orientation on the $\sigma_{i}$, we have

$$
\sigma_{1}+\sigma_{2}+\sigma_{3}+\sigma_{4} \sim 0\left[\text { in } T_{0}(S)\right] .
$$

$\bar{A}_{1}$ and $\sigma_{1}$ are homotopic by a homotopy which rotates the tangent vectors through $180^{\circ}$. Notice that the direction in which the paths are traversed does not change, only the tangent vectors do. A similar homotopy argument shows $\sigma_{3} \sim-\bar{A}_{2}$.

Finally, the induced (boundary) orientation on $\sigma_{4}$ is in the clockwise direction, and one notes that in traversing $\sigma_{4}$ in the clockwise direction, the tangent vector travels once around the origin in the positive direction. Therefore, $\sigma_{4}=\alpha$.

Thus, from (3e) we see that

$$
\bar{C}_{1}=\vec{A}_{2}-\bar{A}_{1}-\alpha .
$$

This enables us to use (3c) to compute the action of $f_{C_{1}}$,

$$
\left(T f_{C_{1}}\right)_{*}(\tilde{\tau})=\tilde{\tau}+\left(C_{1} \times \tilde{\tau}\right) \cdot\left[\bar{C}_{1}\right] .
$$

For any basis element $\tilde{\tau},\left(C_{1} \times \tilde{\tau}\right)=0$ unless $\bar{B}_{1}=\tilde{\tau}$ or $\bar{B}_{2}=\tilde{\tau}$. Now $\left(C_{1} \times B_{2}\right)=1$, and $\left(C_{1} \times B_{1}\right)=-1$. Therefore,

$$
\left(T f_{C_{1}}\right)_{*}\left(\bar{B}_{1}\right)=\bar{B}_{1}+\bar{A}_{1}+\alpha-A_{2}
$$

and

$$
\left(T f_{C_{1}}\right)_{*}\left(\bar{B}_{2}\right)=\bar{B}_{2}+\bar{A}_{2}-\bar{A}_{1}-\alpha .
$$

So the matrix for $\left(T f_{C_{1}}\right)_{*}$ is as claimed.

After taking inverses in Proposition 3.2, we have proved Theorem B, which we now state more explicitly:

Theorem B. The action (2f) of $\operatorname{Mod}\left(I_{g}\right)$ on $n^{\text {th }}$ roots (viewed as elements of $A\left(T_{0}(S)\right)$ ) can be computed for the set $\left\{\hat{r}_{g}, \hat{f}_{A_{1}},{\hat{B_{1}}}_{B_{1}}, \hat{f}_{C_{1}}\right\}$ of generators for $\operatorname{Mod}\left(\Gamma_{g}\right)$. We have

$$
\begin{array}{ll}
\hat{r}_{g} \cdot \lambda=\lambda\left(\begin{array}{ccc}
R^{g-1} & 0 & 0 \\
0 & R^{g-1} & 0 \\
0 & 0 & 1
\end{array}\right) & \hat{f}_{A_{1}} \cdot \lambda=\lambda\left(\begin{array}{ccc}
I & -E & 0 \\
0 & I & 0 \\
0 & 0 & 1
\end{array}\right) \\
\hat{f}_{B_{1}} \cdot \lambda=\lambda\left(\begin{array}{ccc}
I & 0 & 0 \\
E & I & 0 \\
0 & 0 & 1
\end{array}\right) & \hat{f}_{C_{1}} \cdot \lambda=\lambda\left(\begin{array}{ccc}
I & -M & 0 \\
0 & I & 0 \\
0 & -v & 1
\end{array}\right) .
\end{array}
$$




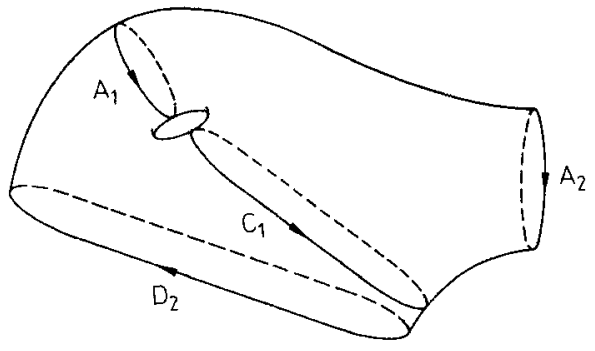

a
Fig. 5a-b

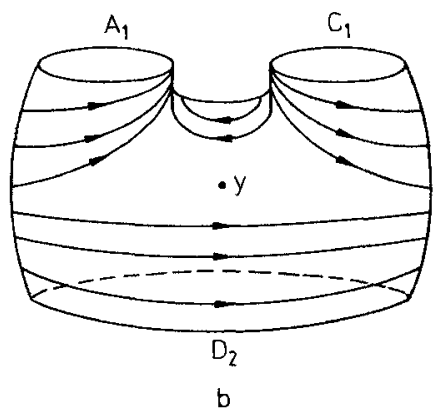

\section{The Kernel of The Action (Theorem C)}

4.1. Recall that $G_{g, n}$ is the subgroup of $\operatorname{Mod}\left(\Gamma_{g}\right)$ which fixes all $n^{\text {th }}$ roots of $K\left(V_{g}\right)$. We have seen that $G_{g, n}$ is a subgroup of finite index, and (Theorem A) elements $\hat{f}$ of $G_{g, n}$ are characterized by the condition $(T f)_{*} \equiv I(\bmod n)$. Of course, since the upper left hand $(2 g \times 2 g)$ corner of the matrix $(T f)_{*}$ is $\varrho(\hat{f})$, if $\hat{f} \in G_{g, n}$ then $\varrho(\hat{f})$ is congruent to $I(\bmod n)$. Here we study the images under the homomorphism $Q$ of the groups $G_{g, n}$ in the symplectic group $\operatorname{Sp}(g, \mathbb{Z})$.

Using our methods, Earle's example, (discussed in Sect. 2.2) takes this form:

Example. Consider the element $g \in \operatorname{Mod}\left(\Gamma_{q}\right)$ given by the composition of the Dehn twists $f_{A_{2}}^{-1}$ and $f_{D_{2}}$, indicated in Fig. 5a

$$
\hat{g}=f_{A_{2}}^{-1} \circ f_{D_{2}} \text {. }
$$

Then $\varrho(\hat{g})=I$, but $g \notin G_{g, 2 g-2}$ for $g \geqq 3$.

One sees this using the method of the proof of Proposition 3.2, using the vector field in Fig. 5b.

One finds that

$$
\hat{g} \cdot \lambda=\lambda \circ\left(\begin{array}{ccc}
I & 0 & 0 \\
0 & I & 0 \\
0 & -w & 1
\end{array}\right),
$$

where $w=\left(w_{1}, \ldots, w_{g}\right)$ has $w_{2}=-2$, all other entries zero. Thus if $g \geqq 3, \hat{g}$ does not fix all the $(2 g-2)^{\text {th }}$ roots, so $\hat{g} \notin G_{g, 2 g-2}$.

Remark. These geometric methods give still another way of noticing that the loop around the origin has order $2 g-2$. A surface of genus $g$ can be decomposed into $(g-1)$ surfaces like that in Fig. 5a, and each has such a vector field with two singularities. Since the whole surface has no boundary, we conclude that $(2 g-2) \alpha=0$.

Let $N_{g, n}$ be the normal subgroup of $\operatorname{Sp}(g, \mathbb{Z})$ of matrices congruent to $I(\bmod n)$. In [14] Mennicke proves the following theorem:

Mennicke's Theorem. $N_{g, n}$ is the smallest normal subgroup of $\operatorname{Sp}(g, \mathbb{Z})$ containing the matrix

$$
\left(\begin{array}{cc}
I & n E \\
0 & I
\end{array}\right)
$$


We already know that the image $\varrho\left(G_{g, n}\right)$ is contained in the congruence subgroup $N_{g, n}$. In fact, those groups are the same.

Theorem $\mathrm{C}$. The image of the group $G_{g, n}$ in the symplectic group equals the congruence subgroup of rank $n$. That is,

$$
\varrho\left(G_{g, n}\right)=N_{g, n} .
$$

Proof. It suffices to show that $\varrho$ maps $G_{g, n}$ onto $N_{g, n}$. By Mennicke's theorem, we need only find $\hat{h} \in G_{g, n}$ with the property that

$$
\varrho(\hat{h})=\left(\begin{array}{cc}
I & n E \\
0 & I
\end{array}\right) .
$$

For $G_{g, n}$ is normal in $\operatorname{Mod}\left(\Gamma_{g}\right)$, and $\varrho$ maps onto the symplectic group, so $\varrho\left(G_{g, n}\right)$ is normal in the symplectic group. The existence of $\hat{h}$ as above would mean that $N_{g, n} \subseteq \varrho\left(G_{g, n}\right)$. Take $\hat{h}=\left(f_{A_{1}}\right)^{n}$. Then it is easy to see that $\hat{h} \in G_{g, n}$, and since we already know that $\varrho\left(f_{A_{1}}\right)=\left(\begin{array}{ll}I & E \\ 0 & I\end{array}\right)$, it follows that $\varrho(\hat{h})=\left(\begin{array}{cc}I & n E \\ 0 & I\end{array}\right)$ and the theorem
is proved.

\section{The Bijection of Theorem 1}

5.1. Definition. Let $B$ be a complex manifold. Denote the loop around the origin in the punctured canonical bundle $K_{0}(B)$ by $\beta$, and the loop around the origin in its dual $K_{0}^{*}(B)$ by $\propto$. Define :

$$
\begin{aligned}
& \Lambda\left(K_{0}(B)\right)=\left\{\lambda: H_{1}\left(K_{0}(B), \mathbb{Z}_{n}\right) \rightarrow \mathbb{Z}_{n}, \lambda \text { a homomorphism, } \lambda(\beta)=1\right\} . \\
& \Lambda\left(K_{0}^{*}(B)\right)=\left\{\lambda: H_{1}\left(K_{0}^{*}(B), \mathbb{Z}_{n}\right) \rightarrow \mathbb{Z}_{n}, \lambda \text { a homomorphism, } \lambda(\alpha)=-1\right\} .
\end{aligned}
$$

We will work only with $B=V_{q}$ or $B=X_{t}$, and $n \mid 2 g-2$. Since $K_{0}\left(X_{t}\right) \subseteq K_{0}\left(V_{g}\right)$, the loop around the origin in $K_{0}\left(X_{t}\right)$ really is the loop around the origin in $K_{0}\left(V_{g}\right)$, thus no confusion will result in denoting them both by $\beta$. Similarly for $\alpha \in K_{0}^{*}\left(V_{g}\right)$ and $\alpha \in K_{0}^{*}\left(X_{2}\right)$. We identify $T_{0}(S)$ with $K_{0}^{*}\left(X_{0}\right)$ [and in 5.2 will make precise an identification of $K_{0}^{*}\left(X_{t}\right)$ with $T_{0}(S)$ ], so our notation also agrees with that of Sect. 2.5 [and $\left.\Lambda\left(T_{0}(S)\right)=\Lambda\left(K_{0}^{*}\left(X_{0}\right)\right)\right]$. We can now make the statement of Theorem 1 more precise.

Theorem 1a. Let $L \rightarrow K\left(V_{g}\right) \rightarrow V_{g}$ be an $n^{\text {th }}$ root of $K\left(V_{g}\right)$. The covering map $p: L_{0} \rightarrow K_{0}\left(V_{g}\right)$ determines a homomorphism in $\Lambda\left(K_{0}\left(V_{g}\right)\right)$. That correspondence induces a bijection between the set of (equivalence classes of) $n^{\text {th }}$ roots of the canonical bundle and the set $A\left(K_{0}\left(V_{g}\right)\right)$.

Theorem 1b. $A$ Hermitian metric on $V_{g}$ induces a bijection between $\Lambda\left(K_{0}\left(V_{\theta}\right)\right)$ and $\Lambda\left(T_{0}(S)\right)$.

The isomorphism is not canonical; we will choose a hermitian metric on $V_{g}$ and construct the bijection of Theorem $1 \mathrm{~b}$ in Sect. 5.2. The proof of Theorem 1a is rather long and will be broken up into several lemmas.

Let Aut $(\tilde{X}, p)$ denote the group of deck transformations of the covering map $p: \tilde{X} \rightarrow X$. 
Lemma 1. If $L$ is an $n^{\text {th }}$ root of $K\left(V_{g}\right)$, and $p: L_{0} \rightarrow K_{0}\left(V_{g}\right)$ is the map of Proposition 2.3, then Aut $\left(L_{0}, p\right)$ is a cyclic group of order $n$, with generator corresponding to $\beta$, the loop around the origin in $\pi_{1}\left(K_{0}\left(V_{g}\right), b\right)$.

Proof. Let $b=(s, \xi)$ be the basepoint in $K_{0}\left(V_{g}\right)$; so $s \in V_{g}$, and $\xi$ is a point in the fibre $\left(\mathbb{C}^{*}\right)$ of $K_{0}\left(V_{g}\right)$ over $V_{g}$. Let $\xi_{i}, i=0,1, \ldots, n-1$ be the $n^{\text {th }}$ roots of $\xi$ (with $\xi_{0}$ the primitive $n^{\text {th }}$ root, and $\left.\arg \xi_{i+1}=\frac{2 \pi}{n}+\arg \xi_{i}\right)$. Every right $\pi_{1}\left(K_{0}\left(V_{g}\right), b\right)$-space automorphism of the fibre is of the form $\left(s, \xi_{i}\right) \rightarrow\left(s, \xi_{i+k}\right)$ where $k$ is an integer $\bmod n$, because of the equivariance condition. Now $\beta$ acts on $p^{-1}(b)$ by $\left(s, \xi_{i}\right) \mapsto\left(s, \xi_{i+1}\right)$, so $\beta$ is a generator $\beta \mapsto 1$ determines an isomorphism of the group of $\pi_{1}\left(K_{0}\left(V_{g}\right), b\right)$ automorphisms of $p^{-1}(b)$ with $\mathbb{Z}_{n}$. Thus, Aut $\left(L_{0}, p\right) \simeq \mathbb{Z}_{n}$.

Remark. From the proof of Lemma 1, it is clear that $\pi_{1}\left(K_{0}\left(V_{q}\right), b\right)$ operates transitively on $p^{-1}(b)$. Thus $\operatorname{Aut}\left(L_{0}, p\right) \simeq \pi_{1}\left(K_{0}\left(V_{g}\right), b\right) / p_{*}\left(\pi_{1}\left(L_{0}, \ell\right)\right)$ where $b=(s, \xi) \in K_{0}\left(V_{q}\right)$ and $\ell \in p^{-1}(b)$. Note that we have fixed an isomorphism $\pi_{1}\left(K_{0}\left(V_{g}\right), b\right) / p_{*}\left(\pi_{1}\left(L_{0}, \ell\right)\right) \simeq \mathbb{Z}_{n}$ which carries $\beta$, the loop around the origin, to 1

Lemma 2. There is a mapping $F$ which assigns an element of $\Lambda\left(K_{0}\left(V_{g}\right)\right.$ to each $n^{\text {th }}$ root of the canonical bundle. $F$ is well-defined on equivalence classes of bundles.

Proof. Given $L$, an $n^{\text {th }}$ root of $K\left(V_{g}\right)$, a covering map $p: L_{0} \rightarrow K_{0}\left(V_{g}\right)$ induces a homomorphism $\pi_{1}\left(K_{0}\left(V_{g}\right), b\right) \rightarrow \mathbb{Z}_{n}$ which we obtain by composition of the quotient map $\pi_{1}\left(K_{0}\left(V_{g}\right), b\right) \rightarrow \pi_{1}\left(K_{0}\left(V_{g}\right), b\right) / p_{*}\left(\pi_{1}\left(L_{0}, \ell\right)\right)$ with the isomorphism fixed in the previous remark. One checks that map factors through the bottom row of the commutative diagram

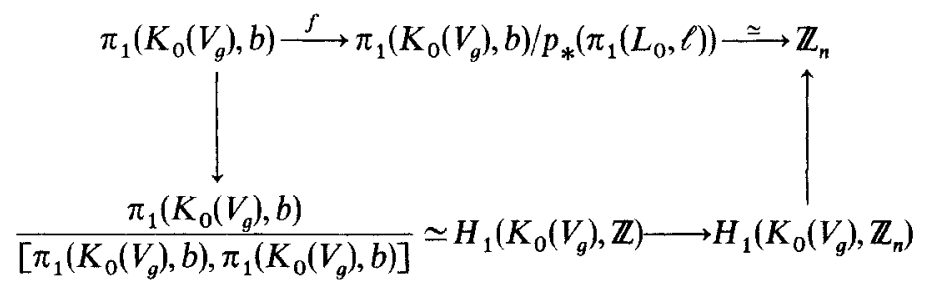

to give $\lambda: H_{1}\left(K_{0}\left(V_{g}\right), \mathbb{Z}_{n}\right) \rightarrow \mathbb{Z}_{n}$. By our choice of isomorphism with $\mathbb{Z}_{n}, \lambda \in \Lambda\left(K_{0}\left(V_{g}\right)\right)$.

The $\lambda$ so constructed does not depend on the choice of the equivalence $L^{\otimes n} \rightarrow K\left(V_{g}\right)$. For if $\Phi: K\left(V_{g}\right) \rightarrow L^{\otimes n}$ and $\Psi: K\left(V_{g}\right) \rightarrow L^{\otimes n}$ determine $\lambda$ and $\lambda^{\prime}$, then $\lambda=\left(\Psi^{-1} \circ \Phi\right)_{*} \circ \lambda^{\prime}$. But $\Psi^{-1} \circ \Phi$ is a holomorphic self-equivalence of the bundle $K\left(V_{g}\right) \rightarrow V_{g}$, and is thus homotopic to the identity by the Corollary of the Lemma in Sect. 2.3.

It is straightforward (by checking commutativity of the appropriate diagram) to verify that $F$ is well defined on equivalence classes.

Lemma 3. There is a mapping $G$ which assigns to each element of $\Lambda\left(K_{0}\left(V_{g}\right)\right)$ a line bundle $L$ which satisfies $L^{\otimes_{n}} \simeq K_{0}\left(V_{g}\right)$.

Proof. Given $\lambda \in K_{0}\left(V_{g}\right)$, choose a base point $b$, and let $f: \pi_{1}\left(K_{0}\left(V_{g}\right), b\right) \rightarrow \mathbb{Z}_{n}$ be defined by the composition $\pi_{1}\left(K_{0}\left(V_{g}\right), b\right) \rightarrow H_{1}\left(K_{0}\left(V_{g}\right), \mathbb{Z}_{n}\right) \stackrel{\lambda}{\rightarrow} \mathbb{Z}_{n}$. Then ker $f$ is a 
subgroup of $\pi_{1}\left(K_{0}\left(V_{g}\right), b\right)$, so by the classification theory for covering spaces (e.g. see $\left[16\right.$, Chap. 8]), there is a covering space $E$ and a point $e \in p^{-1}(b)$ so that $p_{*}\left(\pi_{1}(E, e)\right)=\operatorname{ker} f$. The $n$-cyclic map $p$ is a local homeomorphism, so the complex structure on $K_{0}\left(V_{g}\right)$ lifts to give a manifold structure on $E$ so that $p$ is holomorphic. The covering map $p: E \rightarrow K_{0}\left(V_{g}\right)$ also gives an action of $\pi_{1}\left(K_{0}\left(V_{g}\right), b\right) / p_{*}\left(\pi_{1}(E, e)\right) \simeq \mathbb{Z}_{n}$ on $E$ so that $p\left(v_{1}\right)=p\left(v_{2}\right)$ if and only if $v_{2}=v_{1} \cdot \beta^{k}$, $0 \leqq k \leqq n-1$, where $\beta$ is our generator for $\mathbb{Z}_{n}$.

Our "punctured" line bundles correspond to holomorphic principal $\mathbb{C}^{*}$-bundles, and we can easily pass to line bundles (fibre $\mathbb{C}$ ) by "filling in the punctures". Thus, to complete the proof of the lemma, we show that $E$ has the structure of a holomorphic principal $\mathbb{C}^{*}$ bundle $L_{0}$ (corresponding to a complex line bundle $L$ ). That is, there is a holomorphic map $\phi: \mathbb{C}^{*} \times L_{0} \rightarrow L_{0}$ satisfying

i) $\phi\left(z_{1} z_{2}, v\right)=\phi\left(z_{1}, \phi\left(z_{2}, v\right)\right) \quad \forall v \in L_{0}, z_{1}, z_{2} \in \mathbb{C}^{*}$.

ii) $\phi(1, v)=v \quad \forall v \in L_{0}$.

iii) If $\phi(z, v)=v$ for some $v$, then $z=1$.

iv) The orbit space $L_{0} / \mathbb{C}^{*}$ is a complex manifold $M$ with holomorphic quotient map $\pi: L_{0} \rightarrow M$.

v) There is a cover $\left\{U_{\alpha}\right\}$ of $M$ and local holomorphic sections $s_{\alpha}: U_{\alpha} \rightarrow L_{0}$ such that the $\operatorname{map} h_{\alpha}(p, z)=\phi\left(z, s_{\alpha}(p)\right)$ is a biholomorphic map from $U_{\alpha} \times \mathbb{C}^{*}$ to $\pi^{-1}\left(U_{\alpha}\right)$.

We often write the action $\phi(z, v)=z \cdot v$.

We must also show that $L$ is an $n^{\text {th }}$ root of $K\left(V_{g}\right)$, i.e.

vi) $L^{\otimes n}=K\left(V_{g}\right)$.

The function $G$ of the lemma is $G(\lambda)=L$.

$K_{0}\left(V_{g}\right)$ itself has the structure of a principal $\mathbb{C}^{*}$ bundle [we write $K_{0}\left(=K_{0}\left(V_{g}\right)\right)$ for the remainder of the discussion]. That is, $\psi: \mathbb{C}^{*} \times K_{0} \rightarrow K_{0}$ is an action of $\mathbb{C}^{*}$ on $K_{0}$ satisfying i) through $\mathrm{v}$ ) above and we write $\psi(z, w)=z \cdot w$.

Define $\tilde{p}: \mathbb{C}^{*} \times E \rightarrow \mathbb{C}^{*} \times K_{0}$ by

$$
\tilde{p}(z, v)=\left(z^{n}, p(v)\right) \text {. }
$$

The existence of a mapping $\phi$ satisfying $\phi(1, e)=e$ and making the diagram

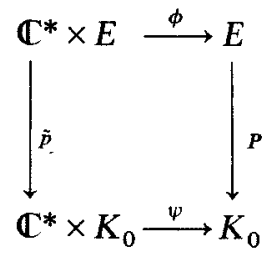

commute is guaranteed by the general lifting theorem from covering space theory, once we check that $(\psi p)_{*}\left(\pi_{1}\left(\mathbb{C}^{*} \times E,(1, e)\right) \leqq p_{*}\left(\pi_{1}(E, e)\right)\right.$. Now $\pi_{1}\left(\mathbb{C}^{*} \times E,(1, e)\right)$ is the product of the fundamental groups of $\mathbb{C}^{*}$ and $E$. The definition of $\tilde{p}$ makes it clear that $(\psi \tilde{p})_{*}\left[\pi_{1}(E, e]=p_{*}\left(\pi_{1}(E, e)\right)\right.$. Also, $(\psi \tilde{p})_{*}$ takes the generator of $\pi_{1}\left(\mathbb{C}^{*}\right)$ (the loop around the origin in $\left.\mathbb{C}^{*} \times\{e\}\right)$ to $\beta^{n} \in p_{*}\left(\pi_{1}(E, e)\right.$ ), so the lift $\phi$ exists.

Recall that lift is defined geometrically as follows : For $(z, v) \in \mathbb{C}^{*} \times E$, let $\tau$ be a path connecting $(1, e)$ with $(z, v)$. Then $(\psi \tilde{p})_{*} \tau$ is a path in $K_{0}$. Lift that path to a path in $E$ starting at $e$, and let $\phi(z, v)$ be the end point of that lift. The proofs that $\phi$ satisfies ii) and iii) are more or less straightforward path lifting arguments using this definition of $\phi$, and are omitted. 
Proof of i). There is a covering map $(z, w, v) \rightarrow\left(z^{n}, w^{n}, p(v)\right)$ from $\mathbb{C}^{*} \times \mathbb{C}^{*} \times E \rightarrow \mathbb{C}^{*} \times \mathbb{C}^{*} \times K_{0}$. Let $\Psi$ be the map $\mathbb{C}^{*} \times \mathbb{C}^{*} \times K_{0} \rightarrow K_{0}$ given by $\Psi(z, w, v)=\psi(z w, v)=\psi(z, \psi(w, v))$. A computation shows that the maps from $\mathbb{C}^{*} \times C^{*} \times E \rightarrow E$ given by $\phi\left(z_{1} z_{2}, v\right)$ and $\phi\left(z_{1}, \phi\left(z_{2}, v\right)\right)$ both cover the map $\Psi$, making the diagram

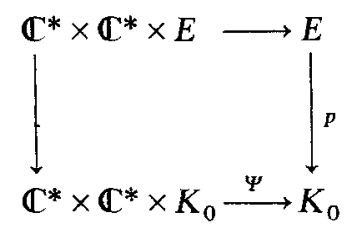

commute. So the two maps differ by a cover transformation; $\phi\left(z_{1}, \phi\left(z_{2}, v\right)\right)=\phi\left(z_{1} z_{2}, v\right) \cdot \beta^{k}$ for all $z_{1}, z_{2} \in \mathbb{C}^{*}$, where $k$ is an integer $\bmod n$. Setting $z_{1}=z_{2}=1$ and using ii), we find that $\beta^{k}$ is the identity, proving i).

Proof of $i v)$. It suffices to show that the equivalence classes of points of $E$ by the action of $\mathbb{C}^{*}$ are the sets $\left\{(\pi \circ p)^{-1}(m)\right\}_{m \in V_{g}}=K_{0} / \mathbb{C}^{*}$. Here, $\pi: K_{0} \rightarrow K_{0} / \mathbb{C}^{*}$ is the quotient map. ,

Suppose $\pi \circ p\left(v_{1}\right)=\pi \circ p\left(v_{2}\right)$. Then $p\left(v_{1}\right) \equiv p\left(v_{2}\right)\left(\bmod \mathbb{C}^{*}\right)$, that is, there exists $z \in \mathbb{C}^{*}$ so that $\psi\left(z, p\left(v_{1}\right)\right)=p\left(v_{2}\right)$. If $z^{1 / n}$ is any $n^{\text {th }}$ root of $z$, $\psi\left(z, p\left(v_{1}\right)\right)=\psi \tilde{p}\left(z, v_{1}\right)=p \phi\left(z^{1 / n}, v_{1}\right)$. So $\phi\left(z^{1 / n}, v_{1}\right) \cdot \beta^{k}=v_{2}$, for some integer $k(\bmod n)$.

Let $z_{0}$ be the $n^{\text {th }}$ root of 1 with $\operatorname{Arg} z_{0}=\frac{2 \pi k}{n}$. Then $\phi\left(z_{0}, \phi\left(z^{1 / n} \cdot v_{1}\right)\right)$ $=\phi\left(z^{1 / n}, v\right) \cdot \beta^{k}=v_{2}$. Thus $v_{2}=\phi\left(z_{0} z^{1 / n}, v_{1}\right)$, so $v_{1} \equiv v_{2} \bmod \mathbb{C}^{*}$. Conversely one can check that if $v_{1} \equiv v_{2} \bmod \mathbb{C}^{*}, \pi \circ p\left(v_{1}\right)=\pi \circ p\left(v_{2}\right)$. The equivalence classes are as claimed, and the quotient map $\pi^{\prime}=\pi \circ p$ is holomorphic.

Proof of $v$ ). (Local triviality) The local triviality of the bundle $K_{0}\left(V_{g}\right)$ gives maps $h_{\alpha}: U_{\alpha} \times \mathbb{C}^{*} \rightarrow \pi^{-1}\left(U_{\alpha}\right)$. We may choose $U_{\alpha}$ connected and simply connected, and check that these lift to $\tilde{h}_{\alpha}$ making the diagram

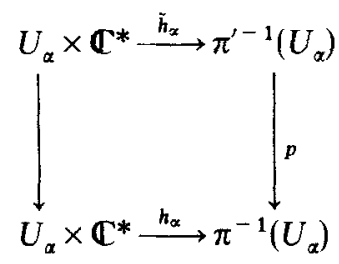

commute. Here $p$ is the restriction of the covering map $p: E \rightarrow K_{0}$ and the unmarked vertical arrow is the covering map $(m, z) \rightarrow\left(m, z^{n}\right)$. $\tilde{h}_{\alpha}$ is a principal $\mathbb{C}^{*}$-bundle isomorphism, giving local trivializations for $L_{0}$.

Proof of vi). The transition functions for $K\left(V_{g}\right)$ are of the form $\kappa_{\beta \alpha}$ where $h_{\beta}^{-1} h_{\alpha}=\left(\mathrm{id} \times \kappa_{\beta \alpha}\right)$. The preceeding diagram then says $\tilde{h}_{\beta}^{-1} \tilde{h}_{\alpha}=\left(\mathrm{id} \times \varrho_{\beta \alpha}\right)$, where $\varrho_{\beta \alpha}$, the transition functions for $L$, satisfy $\varrho_{\beta \alpha}^{n}=\kappa_{\beta \alpha}$. That says $L$ is an $n^{\text {th }}$ root of $K(V g)$.

The proof of Lemma 3 is complete. 
Lemma 4. The mapping $F$ of Lemma 2 is bijective (with inverse $G$ ).

Proof. Given $\lambda \in \Lambda\left(K_{0}\left(V_{g}\right)\right.$, construct $L=G(\lambda)$ as in Lemma 3, so $L_{0}$ covers $K_{0}\left(V_{g}\right)$ and $p_{*}\left(\pi_{1}\left(L_{0}, e\right)\right)=\operatorname{ker} f$. Let $\lambda^{\prime}=F(L)$ [as constructed in diagram (5a)]. Then the diagram

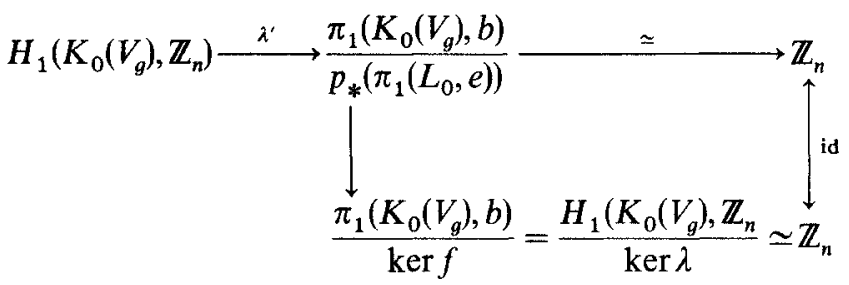

commutes, so $F \circ G$ is the identity on $A\left(K_{0}\left(V_{g}\right)\right)$.

Conversely, let $L$ be an $n^{\text {th }}$ root of $K\left(V_{g}\right), F(L)=\lambda \in \Lambda\left(K_{0}\left(V_{g}\right)\right)$. Construct $L^{\prime}=G(\lambda)$. We complete the proof of the lemma by constructing an equivalence $L \simeq L^{\prime}$, showing $G \circ F$ is the identity on the set of $n^{\text {th }}$ roots. We have covering maps $p: L_{0} \rightarrow K_{0}\left(V_{g}\right)$, and $p^{\prime}: L_{0}^{\prime} \rightarrow K_{0}\left(V_{g}\right)$; let $\ell$ and $\ell^{\prime}$ be the base points in $L_{0}$ and $L_{0}^{\prime}$. By definition, $\operatorname{ker} f=\left(p_{1}\right)_{*}\left(\pi_{1}(L, \ell)\right)$ and by construction, $\left(p_{2}\right)_{*}\left(\pi_{1}\left(L^{\prime}, \ell\right)\right)=\operatorname{ker} f$. Therefore (classification theorem) there is a covering space equivalence $\Phi: L_{0} \rightarrow L_{0}^{\prime}$.

Let $\phi: \mathbb{C}^{*} \times L_{0} \rightarrow L_{0}, \chi: \mathbb{C}^{*} \times L_{0}^{\prime} \rightarrow L_{0}^{\prime}$ and $\psi: \mathbb{C}^{*} \times K_{0} \rightarrow K_{0}$ be the actions which give the principal $\mathbb{C}^{*}$-bundle structures. The following diagram commutes:

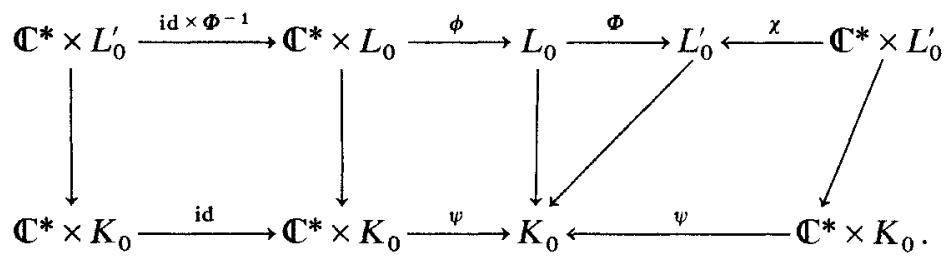

Then

$$
\Phi \circ \phi \circ\left(\mathrm{id} \times \Phi^{-1}\right)=\chi
$$

because both sides of the equality cover $\psi$. Applying $(5 \mathrm{~b})$ to the point $(z, \Phi(v))$ shows that $\Phi(\phi(z, v))=\chi(z, \Phi(v))$. That is, $\Phi$ is a $\mathbb{C}^{*}$-bundle isomorphism, and we are done.

Theorem la is proved.

5.2. By a Hermitian structure on a vector bundle $\pi: L \rightarrow M$, we mean a $C^{\infty}$ function $\langle\rangle:, L \oplus L \rightarrow \mathbb{C}$, such that the restriction $\langle,\rangle_{p}$ to any fibre $\pi^{-1}(p)$ is a Hermitian inner product on that fibre. A Hermitian metric on a manifold $M$ is a Hermitian structure on its tangent bundle. Since the Bers fibre space $F_{g}$ is a bounded domain in $\mathbb{C}^{\mathbf{3 g - 3}}$, it carries a Bergman (hermitian) metric. 
Proposition 5.1. The Bergman hermitian metric on $F_{g}$ induces a Hermitian metric on $V_{g}$. The group $\operatorname{Mod}\left(\Gamma_{g}\right)$ acts as a group of isometries on $V_{g}$. Moreover, the metric induces a Hermitian structure on the canonical bundle $K\left(V_{g}\right)$ and on its dual $K^{*}\left(V_{g}\right)$.

Proof. Since $\bmod \left(\Gamma_{g}\right)$ acts as a group of biholomorphic maps on $F_{g}, \bmod \left(\Gamma_{g}\right)$ is a group of isometries in the Bergman metric. In particular, $\Gamma_{g} \subseteq \bmod \left(\Gamma_{g}\right)$ acts as a group of isometries. The manifold $V_{g}=F_{g} / \Gamma_{g}$ has the quotient metric and $\operatorname{Mod}\left(\Gamma_{g}\right)$ is a group of isometries.

In local coordinates, the Bergman metric on $V_{g}$ is specified by a positive definite hermitian symmetric matrix $\left[g_{i j}\right]$ with $C^{\infty}$ entries $g_{i j}(v)$ where $v=\left(v_{1}, v_{2}, \ldots, v_{m}\right)$ is a local coordinate at $p \in V_{g}$. The metric is, of course, invariantly defined, so under a change of coordinates $V=F(u)$, we have

$$
\left[g_{i j}(u)\right]=[D F] \cdot\left[g_{i j}(v)\right] \cdot\left[\overline{D F}^{t}\right] .
$$

That metric induces a Hermitian structure $g^{i \bar{j}}$ on the dual bundle $T^{*}\left(V_{g}\right)$, where $\left[g^{i j}\right]$ is the inverse of the matrix $\left[g_{i j}\right]$. The canonical line bundle $K\left(V_{g}\right)$ is by definition the determinant of the bundle $T^{*}\left(V_{g}\right)$, so $h^{1 \overline{1}}(v)=\operatorname{det}\left[g^{i \bar{j}}(v)\right]$ determines a Hermitian structure on the line bundle $K\left(V_{g}\right)$. The dual metric $h_{1 \overline{1}}(v)=\operatorname{det}\left[g_{i j}(v)\right]$ is a Hermitian structure on $K^{*}\left(V_{g}\right)$ and we see easily from (5c) that under a change of coordinates $v=F(u)$,

$$
h_{1 \overline{1}}(u)=\operatorname{det}(D F) \cdot h_{1 \overline{1}}(v) \cdot \operatorname{det}\left(\overline{D F}^{t}\right) .
$$

Proposition 5.2. The Hermitian structure $h^{1 \overline{1}}$ on $K\left(V_{g}\right)$ induces a fibre-preserving map $h: K\left(V_{g}\right) \rightarrow K^{*}\left(V_{g}\right)$, which is a $C^{\infty}$ equivalence of the underlying real vector bundles.

Proof. A hermitian structure on a line bundle always induces such a map to its dual. In this case, the explicit form (in local coordinates) is

$$
h(v, \xi)=(v,\langle, \xi\rangle p)=\left(v, h^{11}(v) \cdot \bar{\xi}\right)
$$

Notice that this map is conjugate linear (therefore sense-reversing) on the fibres.

Proposition 5.3. The mapping $h$ of the previous proposition induces a bijection $H: \Lambda\left(K_{0}\left(V_{g}\right)\right) \rightarrow \Lambda\left(K_{0}^{*}\left(V_{g}\right)\right)$.

Proof. The induced map $h_{*}: H_{1}\left(K_{0}\left(V_{g}\right), \mathbb{Z}_{n}\right) \rightarrow H_{1}\left(K_{0}^{*}\left(V_{g}\right), \mathbb{Z}_{n}\right)$ satisfies $h_{*}(\beta)=-\alpha$ (because of the conjugate linearity of $h$ ). If $\lambda \in \Lambda\left(K_{0}\left(V_{g}\right)\right)$, the desired bijection is defined by $H(\lambda)=\lambda \circ h_{*}^{-1}$.

Proposition 5.4. The diffeomorphism $\theta: V_{g} \rightarrow T_{g} \times S$ of Proposition 2.1 induces a diffeomorphism $\tilde{\theta}: K^{*}\left(V_{g}\right) \rightarrow T_{g} \times T(S) . \tilde{\theta}$ is a $C^{\infty}$ equivalence of the (real dimension 2) vector bundles $K^{*}\left(V_{g}\right) \rightarrow V_{g}$ and $T_{g} \times T(S) \rightarrow V_{g}$. The restriction $\tilde{\theta}^{t}: K^{*}\left(X_{t}\right) \rightarrow T(S)$ is a $C^{\infty}$ equivalence.

Proof. $K^{*}\left(X_{t}\right)$ can be identified with the tangent bundle $T\left(X_{t}\right)$, and with that identification, $\tilde{\theta}^{z}$ is the (real) differential of the diffeomorphism $\theta^{t}$. A calculation in local coordinates shows that the maps $\tilde{\theta}^{t}$ "fit together" to give a diffeomorphism 
making the following diagram commute:

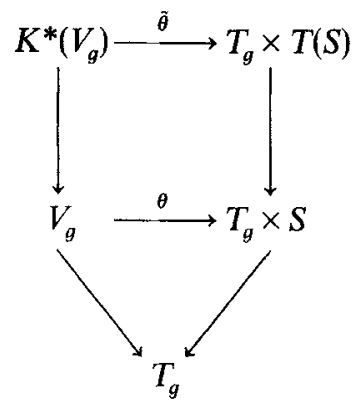

Corollary 1. The inclusions $K_{0}^{*}\left(X_{t}\right) \rightarrow K_{0}^{*}\left(V_{g}\right)$ induce natural isomorphisms on homology.

Proof. The diagram

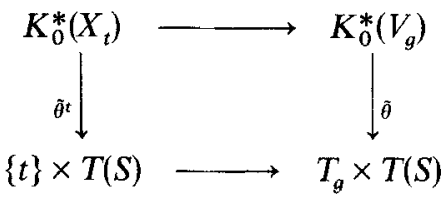

commutes. Since $T_{g}$ is contractible, that says $K_{0}^{*}\left(X_{\vartheta}\right)$ is a deformation retract of $K_{0}\left(V_{g}\right)$, so

$$
i_{t}: H_{1}\left(K_{0}\left(X_{t}\right), \mathbb{Z}_{n}\right) \rightarrow H_{1}\left(K_{0}^{*}\left(V_{g}\right), \mathbb{Z}_{n}\right)
$$

is an isomorphism. Of course, $K_{0}^{*}\left(V_{g}\right)$ can be deformed onto $K_{0}^{*}\left(X_{s}\right)$ for any $s \in T_{g}$, and $K_{0}^{*}\left(X_{s}\right) \simeq K_{0}^{*}\left(X_{t}\right)$ (diffeomorphically).

Corollary 2. The inclusions $K_{0}\left(X_{t}\right) \rightarrow K_{0}\left(V_{g}\right)$ induce natural isomorphisms on homology.

Corollary 3. The isomorphisms of Corollaries 1 and 2 induce bijections

a) $\Lambda\left(K_{0}\left(X_{t}\right)\right) \leftrightarrow \Lambda\left(K_{0}\left(V_{g}\right)\right)$,

b) $\Lambda\left(K_{0}^{*}\left(X_{t}\right)\right) \leftrightarrow \Lambda\left(K_{0}^{*}\left(V_{g}\right)\right)$.

Proposition 5.5. The basis $\left\{\bar{A}_{1}, \ldots, \bar{A}_{g}, B_{1}, \ldots, \bar{B}_{g}, \alpha\right\}$ for $H_{1}\left(K_{0}^{*}\left(X_{0}\right), \mathbb{Z}_{n}\right)$ $=H_{1}\left(T_{0}(S), \mathbb{Z}_{n}\right)$ (fixed in Sect. 2.5) is a basis for $H_{1}\left(K_{0}^{*}\left(V_{g}\right), \mathbb{Z}_{n}\right)$. The basis "restricts" to a homology basis on each fibre $K_{0}^{*}\left(X_{t}\right)$.

Proof. $\left\{\bar{A}_{1}, \ldots, \bar{A}_{g}, \bar{B}_{1}, \ldots, \bar{B}_{g}, \alpha\right\} \subseteq H_{1}\left(K_{0}^{*}\left(V_{g}\right), \mathbb{Z}_{n}\right)$ and form a basis (by Corollary 1$)$. The inverse images under $\theta^{t}: K_{0}^{*}\left(X_{t}\right) \rightarrow K_{0}^{*}\left(X_{0}\right)=T_{0}(S)$ of the basis elements gives a homology basis for each punctured line bundle $K_{0}^{*}\left(X_{t}\right)$. The deformation retraction of $K_{0}^{*}\left(V_{g}\right)$ onto $K_{0}^{*}\left(X_{0}\right)$ maps $\left(\tilde{\theta}^{t}\right)^{-1}(\gamma) \rightarrow \gamma$. So $\left(\tilde{\theta}^{t}\right)^{-1}(\gamma)$ and $\gamma$ are homotopic (thus homologous) in $K_{0}^{*}\left(V_{g}\right)$.

Notice that the map $\left(\tilde{\theta}^{t}\right)^{-1}$ induces a bijection between $\Lambda\left(T_{0}(S)\right)$ and $\Lambda\left(K_{0}^{*}\left(X_{t}\right)\right)$. This fact, together with Corollary $3 \mathrm{~b}$, completes the proof of Theorem $1 \mathrm{~b}$.

Remark. The above choice of homology basis on $K_{0}^{*}\left(V_{g}\right)$ automatically determines bases $\left\{h_{*}^{-1}\left(\bar{A}_{1}\right), h_{*}^{-1}\left(\bar{A}_{2}\right), \ldots, h_{*}^{-1}\left(\bar{B}_{g}\right), \alpha\right\}$ on $H_{1}\left(K_{0}\left(V_{g}\right), \mathbb{Z}_{n}\right)$ and $H_{1}\left(K_{0}\left(X_{0}\right), \mathbb{Z}_{n}\right)$. Hereafter, these bases should be regarded as fixed. 
With the choice of the basis as in Proposition 5.5, the elements of $A\left(K_{0}^{*}\left(X_{t}\right)\right)$ and $A\left(K_{0}^{*}\left(V_{g}\right)\right)$ can be represented as vectors in $\left(\mathbb{Z}_{n}\right)^{2 g+1}$ with final entry -1 [as we saw with $A\left(T_{0}(S)\right]$ at the end of Sect. 2.5). Similarly, we represent the elements of $A\left(K_{0}\left(X_{t}\right)\right)$ and $\Lambda\left(K_{0}\left(V_{q}\right)\right)$ as vectors in $\left(\mathbb{Z}_{n}\right)^{2 g+1}$ with final entry 1 . Let $f \in \operatorname{Diff}^{+}(S)$. The map $\left(K^{*} \hat{f}\right): H_{1}\left(K_{0}^{*}\left(V_{g}\right), \mathbb{Z}_{n}\right) \rightarrow H_{1}\left(K_{0}^{*}\left(V_{g}\right), \mathbb{Z}_{n}\right)$ can be represented by a $(2 g+1) \times(2 g+1)$ matrix with entries in $\mathbb{Z}_{n}$.

As immediate corollaries of Theorem $1 \mathrm{~b}$ and its proof we have:

Corollary 1. Each of the sets $A\left(K_{0}\left(V_{g}\right)\right)$ and $A\left(K_{0}^{*}\left(V_{g}\right)\right)$ is in 1-1 correspondence with the $n^{\text {th }}$ roots of $K\left(V_{g}\right)$. All three sets are finite sets of order $n^{2 g}$.

Corollary 2. Every $n^{\text {th }}$ root of $K\left(V_{g}\right)$ restricts to an $n^{\text {th }}$ root of $K\left(X_{t}\right)$ for each $t \in T_{g}$, and every $n^{\text {th }}$ root of $K\left(X_{t}\right)$ extends uniquely to an $n^{\text {th }}$ root of $K\left(V_{g}\right)$.

\section{Proof of Equivariance in Theorem 1}

Theorem 1c. The bijection between $n^{\text {th }}$ roots of $K\left(V_{g}\right)$ (with the action of $\operatorname{Mod}\left(\Gamma_{g}\right)$ as in the corollary of Proposition 2.4) and $\Lambda\left(T_{0}(S)\right.$ ) (with the action of Proposition 2.6) is an equivariant bijection.

Lemma 1. If $h: K\left(V_{g}\right) \rightarrow K\left(V_{g}\right)$ is the map constructed in Proposition 5.2, the diagram

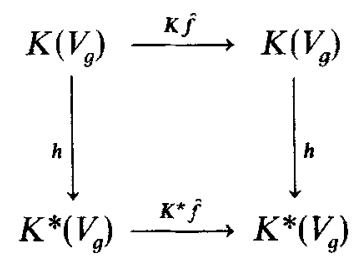

commutes for $\hat{f} \in \operatorname{Mod}\left(\Gamma_{g}\right)$.

Proof. The maps $K \hat{f}$ and $K^{*} \hat{f}$ are defined in Sect. 2.4. The proof is a calculation in local coordinates; the essential fact is that $\hat{f}$ is an isometry in the metric of Proposition 5.1. [A mapping $K\left(V_{g}\right) \rightarrow K^{*}\left(V_{g}\right)$ arising from an arbitrary metric will not generally behave nicely with respect to the "differential" and "codifferential".]

Proposition 6.1. $\operatorname{Mod}\left(\Gamma_{g}\right)$ acts on $\left.\Lambda\left(K_{0} V_{g}\right)\right)$ and on $\Lambda\left(K_{0}^{*}\left(V_{g}\right)\right)$ by the rules

$$
f \cdot \lambda=\lambda \circ\left(K \hat{f}^{-1}\right)_{*}
$$

and

$$
\hat{f} \cdot \lambda^{\prime}=\lambda^{\prime} \circ\left(K^{*} \hat{f}^{-1}\right)_{*},
$$

where $\left(K \hat{f}^{-1}\right)_{*}$ and $\left(K^{*} \hat{f}^{-1}\right)_{*}$ denote the induced maps on homology $(\bmod n)$. Moreover, those actions are the same under the identification of Proposition 5.3.

Proof. The proof of the first statement mimics that of Proposition 2.6, and is therefore omitted. Lemma 1 implies that $\left(K^{*} \hat{f}^{-1}\right)_{*}=h_{*} \circ\left(K \hat{f}^{-1}\right)_{*} \circ h_{*}^{-1}$. With that observation, one checks easily that $H(\hat{f} \cdot \lambda)=\hat{f} \cdot H(\lambda)$, where $H$ is the bijection of Proposition 5.3, to prove the second statement. 
Proposition 6.2. If $\hat{f} \in \operatorname{Mod}\left(\Gamma_{g}\right), \hat{f}: V_{g} \rightarrow V_{g}, L$ an $n^{\text {th }}$ root of $K\left(V_{g}\right)$ and $F(L)=\lambda \in \Lambda\left(K_{0}\left(V_{g}\right)\right)$, and $\lambda^{\prime}=F(\hat{f} \cdot L)$, then

$$
\lambda^{\prime}=F(\hat{f} \cdot L)=\hat{f} \cdot(F(L))=\hat{f} \cdot \lambda,
$$

where $\hat{f} \cdot L$ and $\hat{f} \cdot \lambda$ were defined in $(2 \mathrm{~d})$ and $(6 \mathrm{~b})$ respectively, and $F$ is the correspondence in Sect. 5.1 (Lemma 2).

Proof. From the diagram (2c) we can compare $\lambda \in \Lambda\left(K_{0}\left(V_{g}\right)\right)$ corresponding to the covering map $p$ and $\lambda^{\prime} \in \Lambda\left(K_{0}\left(V_{g}\right)\right)$ corresponding to the covering map $q$. [We can use $q$ to compute $\lambda^{\prime}$ since we proved in Lemma 2 of Sect. 5.1 that all isomorphisms of $L^{\otimes n}$ with $K\left(V_{g}\right)$ give the same element of $\Lambda\left(K_{0}\left(V_{g}\right)\right)$.]

Let $x=(\hat{f}(v), \xi)$ be a basepoint in $\left(\hat{f}^{-1}\right)^{*} L_{0}$. Let $\gamma \in \pi_{1}\left(K_{0}\left(V_{g}\right), x\right)$, and using the covering map $q$, lift it to a path $\tilde{y}$ in the covering space, starting at $\tilde{x}$. Denote the endpoint of the lift by $\tilde{y}$. Since $\tilde{x}$ and $\tilde{y}$ are both elements of $q^{-1}(x)$, we have $\tilde{x}=\left(\hat{f}(v), \xi_{i}\right), \tilde{y}=\left(\hat{f}(v), \xi_{j}\right)$ and $\lambda^{\prime}(\gamma)=j-i=k \in \mathbb{Z}_{n}$.

We have

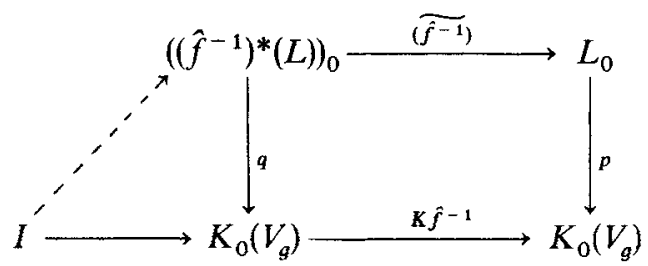

where $\left(\widetilde{\hat{f}^{-1}}\right)$ is the homeomorphism induced by the pullback. Now $\left(K \hat{f}^{-1}\right) \circ \gamma$ is a path in $\pi_{1}\left(K_{0}(V), K \hat{f}^{-1}(x)\right)$. Using $p$, we lift it to a path $\widetilde{K \hat{f}^{-1}} \circ \gamma$ in $L_{0}$, starting at the point $\left(\hat{f}^{-1}\right)(\tilde{x})$. Since the path lifting starting at a given point is unique, $\left(\hat{f}^{-1}\right)(\tilde{x})$ and $\left(\hat{f}^{-1}\right)(\tilde{y})$ are the initial and terminal points of $\widehat{K f}^{-1} \circ \gamma$ (both are elements of $p^{-1}\left(K \hat{f}^{-1}(x)\right)$ and are therefore of the form $\left(v, \eta_{\ell}\right)$ and $\left(v, \eta_{m}\right)$. Thus $\lambda\left(K \hat{f}^{-1} \circ \gamma\right)=m-\ell \in \mathbb{Z}_{n}$.

$\lambda^{\prime}(\alpha)=\lambda\left(\hat{f}^{-1}(\alpha)\right)=1$ because $K \hat{f}^{-1}: \alpha \rightarrow \widetilde{\hat{f}^{-1}}(\alpha)$, so both homotopy classes correspond to the loop around the origin in homology. We see that if $\tilde{\alpha}$ starts at $\left(f(v), \xi_{i}\right)$, it ends at $\left(f(v), \xi_{i+1}\right)$ and if $\vec{f}^{-1}(\tilde{\alpha})=\bar{f}^{-1}(\alpha)$ starts at $\left(v, \eta_{\ell}\right)$ it ends at $\left(v, \eta_{\ell+1}\right)$. Therefore, $m-\ell=k$, so $\lambda\left(K \hat{f}^{-1} \circ \gamma\right)=k$.

$K f^{-1}$ induces an isomorphism

$$
\pi_{1}\left(K_{0}\left(V_{g}\right), x\right) \simeq \pi_{1}\left(K_{0}\left(V_{g}\right), K \hat{f}^{-1}(x)\right) .
$$

So we have $\lambda^{\prime}(\gamma)=k=\lambda\left(\widehat{K \hat{f}^{-1}} \circ \gamma\right)$, where every element of $\pi_{1}\left(K_{0}\left(V_{g}\right), K \hat{f}^{-1}(x)\right)$ is $K \hat{f}^{-1} \circ \gamma$ for some $\gamma$. That is, the diagram

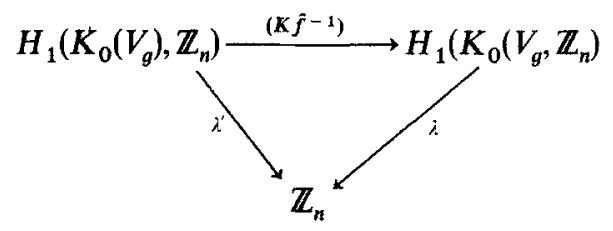

commutes. That says $\lambda^{\prime}=\lambda \circ\left(K \hat{f}^{-1}\right)_{*}=f \cdot \lambda$, as desired. 
Proposition 6.3. The map $T \hat{f}=\tilde{\theta} \circ K^{*}(\hat{f}) \circ \tilde{\theta}^{-1}$ is a fibre-preserving diffeomorphism from $T_{g} \times T(S)$ to itself, which preserves the real vector bundle structure on $T_{g} \times T(S) \rightarrow V_{g} . T \hat{f}_{t}: T(S) \rightarrow T(S)$, the restriction to the fibre over $t \in T_{g}$, is the real differential of the map $\theta^{t} \hat{f}_{t}\left(\theta^{t}\right)^{-1}: S \rightarrow S$. (The diagram

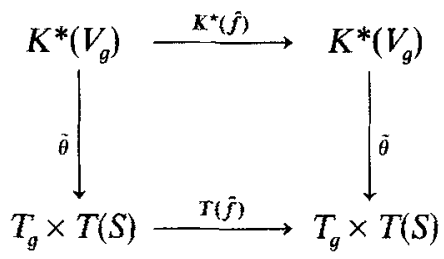

commutes.)

The proof is a calculation using the definitions of the maps and the chain rule.

Recalling the remark following Proposition 5.5, we see that with respect to the basis $\left\{\bar{A}_{1}, \ldots, \bar{A}_{g}, \quad \bar{B}_{1}, \ldots, \bar{B}_{g}, \alpha\right\}, \quad\left(K^{*} f\right)_{*}$ and $(\hat{T f})_{*}$ are represented by $(2 g+1) \times(2 g+1)$ matrices $(\bmod n)$. The action $(6 \mathrm{c})$ can be described by matrix multiplication. [Caution: The reader should remember that we already had a matrix representation for $(T f)_{*}$ and therefore for the action (2d), fixed in Sect. 2.5.]

Proposition 6.4. Let $f \in \operatorname{Diff}^{+}(S)$. The matrix representations $(\bmod n)$ of $\left(K^{*} \hat{f}\right)_{*}$ and $(T f)_{*}$ with respect to the bases we have fixed are the same.

Proof. From Propositions 5.5 and 6.3, it is clear that the matrices of $(T \hat{f})_{*}$ and $\left(K^{*} f\right)_{*}$ are the same (with respect to our fixed bases). $\hat{f}: V_{g} \rightarrow V_{g}$ restricts to $\hat{f}_{t}: X_{t} \rightarrow X_{\hat{f}(t)}$, a conformal mapping. Applying Proposition 6.3 for fixed $t \in T_{g}$ shows that the matrix of $\left(K^{*} \hat{f}\right)_{*}$ is the same as the matrix of $\left(T \hat{f}_{t}\right)_{*}$. But $T \hat{f}_{t}$ is the real differential of $\theta^{t} \circ \hat{f}^{t} \circ\left(\theta^{t}\right)^{-1}: S \rightarrow S$, which is isotopic to $f$. Thus, $\left(T \hat{f_{t}}\right)_{*}=(T f)_{*}$.

Remark. We have seen that an $n^{\text {th }}$ root is determined once it is determined at a single point $t \in T_{g}$. Thus, it is not surprising that we need only the information about the action on one fibre, as the proposition shows.

The proof of Theorem 1 is now complete. To summarize the argument, we have established the three bijections:

1. $n^{\text {th }}$ roots of $K\left(V_{g}\right) \leftrightarrow A\left(K_{0}\left(V_{g}\right)\right)$,

2. $A\left(K_{0}\left(V_{g}\right)\right) \leftrightarrow \Lambda\left(K_{0}^{*}\left(V_{g}\right)\right)$,

3. $\Lambda\left(K_{0}^{*}\left(V_{g}\right)\right) \leftrightarrow \Lambda\left(T_{0}(S)\right)$,

and shown that $\operatorname{Mod}\left(\Gamma_{g}\right)$ acts on each of the four sets. The bijections 1, 2, 3 are equivariant, by Propositions 6.2, 6.1, and 6.4, respectively.

Acknowledgements. Most of these results are contained in the author's Cornell University thesis [18]. It is a pleasure to acknowledge and thank Clifford $\mathrm{J}$. Earle for numerous suggestions and guidance at every stage of its preparation. The author also thanks Andrew Sommese and John Hubbard for several helpful discussions.

\section{References}

1. Auslander, L., Green, L., Hahn, F.: Flows on homogeneous spaces. Ann. Math. Studies, No. 53. Princeton: Princeton University Press 1963

2. Bers, L.: Fiber spaces over Teichmüller spaces. Acta Math. 130, 89-126 (1973) 
3. Bers, L. : Uniformization, moduli, and Kleinian groups. Bull. London Math. Soc. 4, 257-300 (1972)

4. Earle, C.J.: Families of Riemann surfaces and Jacobi varieties. Ann. Math. 107, 255-286 (1978)

5. Earle, C.J.: Roots of the canonical divisor class over Teichmuiller space (preprint)

6. Earle, C.J.: Teichmüller theory. In : Discrete groups and automorphic functions. Harvey, W.J., ed. pp. 143-161. London: Academic Press 1977

7. Ehresmann, C.: Les connexions infinitesimals dans un espace fibre differentiable. Colloque de Topologie, Bruxelles, pp. 29-55 (1950)

8. Griffiths, P., Harris, J.: Principles of algebraic geometry. New York: Wiley 1978

9. Gunning, R.: Riemann surfaces and generalized theta functions. Berlin, Heidelberg, New York: Springer 1976

10. Keen, L.: On Fricke moduli. In : Advances in the theory of Riemann surfaces. Ahlfors et al., L., eds. Ann. Math. Studies, No. 66, pp. 205-224. Princeton: Princeton University Press 1971

11. Lickorish, W.B.R.: A finite set of generators for the homeotopy group of a 2-manifold. Proc. Camb. Phil. Soc. 62, 769-778 (1964)

12. Lickorish, W.B.R.: Corrigendum: a finite set of generators for the homeotopy group of a 2-manifold. Proc. Camb. Phil. Soc. 62, 679-681 (1966)

13. Macbeath, A.M.: Topological background. In: Discrete groups and automorphic functions. Harvey, W.J., ed. pp. 1-46. London: Academic Press 1977

14. Mennicke, Jens L.: Zur Theorie der Siegelschen Modulgruppe. Math. Ann. 159, 115-129 (1965)

15. Munkres, J.: Topology. Englewood Cliffs: Prentice Hall 1975

16. Mumford, D.: Abelian quotients of the Teichmüller modular group. J. Analyse Math. 18, 227-244 (1967)

17. Siegel, C.L.: Topics in complex function theory, Vol. II. New York: Wiley 1971

18. Sipe, P.L.: Roots of the canonical bundle of the universal Teichmüller curve. Thesis, Cornell University, 1979

Received December 3, 1981 